\title{
Dynamics of Collective Decoherence and Thermalization
}

\author{
M. Merkli* G. P. Berman ${ }^{\dagger}$ I. M. Sigal ${ }^{\ddagger}$
}

November 1, 2018

\begin{abstract}
We analyze the dynamics of $N$ interacting spins (quantum register) collectively coupled to a thermal environment. Each spin experiences the same environment interaction, consisting of an energy conserving and an energy exchange part.

We find the decay rates of the reduced density matrix elements in the energy basis. We show that if the spins do not interact among each other, then the fastest decay rates of off-diagonal matrix elements induced by the energy conserving interaction is of order $N^{2}$, while that one induced by the energy exchange interaction is of the order $N$ only. Moreover, the diagonal matrix elements approach their limiting values at a rate independent of $N$. For a general spin system the decay rates depend in a rather complicated (but explicit) way on the size $N$ and the interaction between the spins.

Our method is based on a dynamical quantum resonance theory valid for small, fixed values of the couplings. We do not make Markov-, Born- or weak coupling (van Hove) approximations.
\end{abstract}

\section{Introduction}

Description of the problem. We consider a qubit register of size $N$ whose Hamiltonian is of the form

$$
H_{\mathrm{S}}=\sum_{i, j=1}^{N} J_{i j} S_{i}^{z} S_{j}^{z}+\sum_{j=1}^{N} B_{j} S_{j}^{z},
$$

where the $J_{i j}$ are pair interaction constants that can take positive or negative values, and $B_{j} \geq 0$ is an effective magnetic field at the location of spin $j\left(B_{j}=\frac{\hbar}{2} \gamma B_{j}^{z}\right.$, where

${ }^{*}$ Department of Mathematics and Statistics, Memorial University of Newfoundland, St. John's, NL, Canada A1C 5S7; Supported by NSERC under grant 205247; Email: merkli@math.mun.ca; URL: http://www.math.mun.ca/ merkli/

${ }^{\dagger}$ Theoretical Division and CNLS, MS B213, Los Alamos National Laboratory, Los Alamos, NM 87545, USA; Supported by the NNSA of the U.S. DOE at LANL under Contract No. DE-AC5206NA25396; Email: gpb@lanl.gov

${ }^{\ddagger}$ Department of Mathematics, University of Toronto, Toronto, ON, Canada M5S 2E4; Supported by NSERC under grant NA 7901; Email: im.sigal@utoronto.ca; URL: http://www.math.toronto.edu/sigal/ 
$\hbar$ is the Planck constant, $\gamma$ is the value of the electron gyromagnetic ratio and $B_{j}^{z}$ is an inhomogeneous magnetic field, oriented in the positive $z$ direction). Also,

$$
S^{z}=\left[\begin{array}{cc}
1 & 0 \\
0 & -1
\end{array}\right]
$$

is the Pauli spin $1 / 2$ operator; $S_{j}^{z}$ is the matrix $S^{z}$ acting nontrivially only on the $j$-th spin. The environment $\mathrm{R}$ is modelled by a bosonic thermal reservoir whose Hamiltonian is

$$
H_{\mathrm{R}}=\int_{\mathbb{R}^{3}} a^{*}(k)|k| a(k) \mathrm{d}^{3} k,
$$

where $a^{*}(k)$ and $a(k)$ are the usual bosonic creation and annihilation operators satisfying the canonical commutation relations $\left[a(k), a^{*}(l)\right]=\delta(k-l)$. It is understood that we consider $\mathrm{R}$ in the thermodynamic limit of infinite volume, fixed temperature $T=1 / \beta>0$, in a phase without Bose-Einstein condensate.

We consider a collective coupling: the distance between the $N$ qubits is smaller than the correlation length of the reservoir and consequently each qubit feels the same interaction with the latter. The collective interaction between $\mathrm{S}$ and $\mathrm{R}$ is given by the operator

$$
v=\lambda_{1} v_{1}+\lambda_{2} v_{2}=\lambda_{1} \sum_{j=1}^{N} S_{j}^{z} \otimes \phi\left(g_{1}\right)+\lambda_{2} \sum_{j=1}^{N} S_{j}^{x} \otimes \phi\left(g_{2}\right) .
$$

Here, $\phi(g)$ is the field operator smoothed out with a form factor (coupling function) $g=g(k), k \in \mathbb{R}^{3}$, see (B.1) in Appendix B. The coupling constants $\lambda_{1}$ and $\lambda_{2}$ measure the strengths of the energy conserving (position-position) coupling, and the energy exchange (spin flip) coupling, respectively. Spin-flips are implemented by the $S_{j}^{x}$ in (1.4), representing the Pauli matrix

$$
S^{x}=\left[\begin{array}{ll}
0 & 1 \\
1 & 0
\end{array}\right]
$$

acting on the $j$-th factor of $\mathcal{H}_{\mathrm{S}}$. The total Hamiltonian takes the form

$$
H=H_{\mathrm{S}}+H_{\mathrm{R}}+v
$$

The dynamics of a density matrix $\rho_{t}$ of the system $\mathrm{S}+\mathrm{R}$ is governed by the Liouville-von Neumann equation

$$
\frac{\mathrm{d}}{\mathrm{d} t} \rho_{t}=-\mathrm{i}\left[H, \rho_{t}\right]
$$

with initial condition $\left.\rho_{t}\right|_{t=0}=\rho_{0}$. The solution to the Liouville-von Neumann equation is given by $\rho_{t}=\mathrm{e}^{-\mathrm{i} t H} \rho_{0} \mathrm{e}^{\mathrm{i} t H}$. We are interested only in information on the subsystem $\mathrm{S}$, so we trace out the degrees of freedom of $\mathrm{R}$. The state of $\mathrm{S}$ is given by the reduced density matrix

$$
\bar{\rho}_{t}=\operatorname{Tr}_{\mathrm{R}}\left(\mathrm{e}^{-\mathrm{i} t H} \rho_{0} \mathrm{e}^{\mathrm{i} t H}\right),
$$

where $\rho_{0}$ is the initial density matrix of the coupled system, and $\operatorname{Tr}_{\mathrm{R}}$ is the partial trace over the degrees of freedom of the reservoir. The operator $\bar{\rho}_{t}$ acts on the Hilbert space $\mathcal{H}_{\mathrm{S}}=\mathbb{C}^{2} \otimes \cdots \otimes \mathbb{C}^{2}=\mathbb{C}^{2^{N}}$ of $\mathrm{S}$ only. 
Our goal is to analyze the time evolution of matrix elements of the reduced density matrix (1.7) in the energy basis, which plays a special role in quantum information theory. The energy basis consists of eigenvectors $\varphi_{\underline{\sigma}}$ of $H_{\mathrm{S}}$, indexed by spin configurations

$$
\underline{\sigma}=\left\{\sigma_{1}, \ldots, \sigma_{N}\right\} \in\{+1,-1\}^{N}, \quad \varphi_{\underline{\sigma}}=\varphi_{\sigma_{1}} \otimes \cdots \otimes \varphi_{\sigma_{N}} .
$$

Here,

$$
\varphi_{+}=\left[\begin{array}{l}
1 \\
0
\end{array}\right], \quad \varphi_{-}=\left[\begin{array}{l}
0 \\
1
\end{array}\right]
$$

so that

$$
H_{\mathrm{S}} \varphi_{\underline{\sigma}}=E(\underline{\sigma}) \varphi_{\underline{\sigma}} \quad \text { with } \quad E(\underline{\sigma})=\sum_{i, j=1}^{N} J_{i j} \sigma_{i} \sigma_{j}+\sum_{j=1}^{N} B_{j} \sigma_{j} .
$$

We denote the reduced density matrix elements as

$$
\left[\bar{\rho}_{t}\right]_{\underline{\sigma}, \underline{\tau}}=\left\langle\varphi_{\underline{\sigma}}, \bar{\rho}_{t} \varphi_{\underline{\tau}}\right\rangle .
$$

The dynamics of the register alone (without coupling to the environment) is given by $\bar{\rho}_{t}=\mathrm{e}^{-\mathrm{i} t H_{\mathrm{S}}} \bar{\rho}_{0} \mathrm{e}^{\mathrm{i} t H_{\mathrm{S}}}$, where $\bar{\rho}_{0}=\operatorname{Tr}_{\mathrm{R}}\left(\rho_{0}\right)$, so matrix elements of $\bar{\rho}_{t}$ have the time dependence

$$
\left[\bar{\rho}_{t}\right]_{\underline{\sigma}, \underline{\tau}}=\mathrm{e}^{\mathrm{i} t\{E(\underline{\tau})-E(\underline{\sigma})\}}\left[\bar{\rho}_{0}\right]_{\underline{\sigma}, \underline{\tau}} .
$$

We view the energy differences

$$
e(\underline{\sigma}, \underline{\tau}):=E(\underline{\sigma})-E(\underline{\tau})=\sum_{i, j=1}^{N} J_{i j}\left(\sigma_{i} \sigma_{j}-\tau_{i} \tau_{j}\right)+\sum_{j=1}^{N} B_{j}\left(\sigma_{j}-\tau_{j}\right)
$$

as being eigenvalues of the Liouville operator

$$
L_{\mathrm{S}}=H_{\mathrm{S}} \otimes \mathbb{1}-\mathbb{1} \otimes H_{\mathrm{S}}
$$

acting on the doubled space

$$
\mathcal{H}_{\mathrm{S}} \otimes \mathcal{H}_{\mathrm{S}}=\left(\mathbb{C}^{2} \otimes \mathbb{C}^{2}\right) \otimes \cdots \otimes\left(\mathbb{C}^{2} \otimes \mathbb{C}^{2}\right)
$$

where the $j$-th pair $\mathbb{C}^{2} \otimes \mathbb{C}^{2}$ is the doubled space of the $j$-th qubit.

Discussion of main results. In the resonance approach used in this work, we examine the influence of the interaction (1.4) on the free dynamics (1.12) for small coupling parameters $\lambda_{1}, \lambda_{2}$. Under the perturbation, the phase factors $e=E(\underline{\tau})-E(\underline{\sigma})$ in (1.12) become complex resonance energies, $\varepsilon_{e}=\varepsilon_{e}\left(\lambda_{1}, \lambda_{2}\right) \in \mathbb{C}$. The latter encode properties of irreversibility of the reduced dynamics of $S$ (decay of observables and matrix elements - the dynamics of the entire system $\mathrm{S}+\mathrm{R}$ is unitary, by contrast). We consider the regime where the resonance energies $\varepsilon_{e}\left(\lambda_{1}, \lambda_{2}\right)$ do not overlap as the perturbation is switched on, so that each resonance energy can be followed separately. This means that the coupling parameters must be small with respect to the gap between 
differences of energies of $H_{\mathrm{S}}$, see condition (A1) in Section 2 below. ${ }^{1}$ We make as well a technical assumption (A2) on the regularity of form factors $g_{1}$ and $g_{2}$ which we explain in Section 2.

Dynamics of S. Our first result is a detailed description of the evolution of the reduced density matrix elements (and hence of all observables). Set

$$
\left.\left\langle\left\langle\bar{\rho}_{\infty}\right]_{\underline{\sigma}, \underline{\tau}}\right\rangle\right\rangle=\lim _{T \rightarrow \infty} \frac{1}{T} \int_{0}^{T}\left[\bar{\rho}_{t}\right]_{\underline{\sigma}, \underline{\tau}} \mathrm{d} t .
$$

We show in Theorem 2.1 that this limit exists, and that for all $t \geq 0$,

$$
\begin{aligned}
{\left[\bar{\rho}_{t}\right]_{\underline{\sigma}, \underline{\tau}}-\left\langle\left\langle\left[\bar{\rho}_{\infty}\right]_{\underline{\sigma}, \underline{\tau}}\right\rangle\right\rangle=} & \sum_{\substack{\left\{e, s: \varepsilon_{e}^{(s)} \neq 0\right\}\\
}} \mathrm{e}^{\mathrm{i} t \varepsilon_{e}^{(s)}\left[\sum_{\underline{\sigma}^{\prime}, \underline{\tau}^{\prime}}^{*} w_{\underline{\sigma}, \underline{\tau} ; \underline{\sigma}^{\prime}, \underline{\tau}^{\prime}}^{\varepsilon_{\varepsilon}^{(s)}}\left[\bar{\rho}_{0}\right]_{\underline{\sigma}^{\prime}, \underline{\tau}^{\prime}}+O\left(\lambda_{1}^{2}+\lambda_{2}^{2}\right)\right]} \\
& +O\left(\left(\lambda_{1}^{2}+\lambda_{2}^{2}\right) \mathrm{e}^{-\omega^{\prime} t}\right)
\end{aligned}
$$

where $\operatorname{Im} \varepsilon_{e}^{(s)} \geq 0$ and $\omega^{\prime}$ satisfies $2 \max \left\{\operatorname{Im} \varepsilon_{e}^{(s)}\right\} \leq \omega^{\prime}<\tau$, with $\tau>0$ a constant depending on the regularity of $g_{1}, g_{2}$ (see Condition (A2) in Section 2, and also [16]). The $*$ in the sum (1.17) means that we sum only over configurations $\underline{\sigma}^{\prime}, \underline{\tau}^{\prime}$ such that $e\left(\underline{\sigma}^{\prime}, \tau^{\prime}\right)=-e$. The coefficents $w$ are overlaps of resonance eigenstates (see Section $2.1)$, which vanish unless $e(\underline{\sigma}, \underline{\tau})=-e$, in which case they are $O(1)$ in $\lambda_{1}, \lambda_{2}$. The $\varepsilon_{e}^{(s)}$ are eigenvalues of a certain explicit operator $K\left(\omega^{\prime}\right)$, a "spectrally deformed Liouville operator" (see Section 3.1). They have the expansion

$$
\varepsilon_{e}^{(s)}=e+\delta_{e}^{(s)}+O\left(\lambda_{1}^{4}+\lambda_{2}^{4}\right),
$$

where the label $s=1, \ldots, \nu(e)$ indexes the splitting of the eigenvalue $e$ of $L_{\mathrm{S}}$, having multiplicity $d(e)$, into $\nu(e) \leq d(e)$ distinct resonance energies. The lowest order corrections $\delta_{e}^{(s)}$ satisfy

$$
\delta_{e}^{(s)}=O\left(\lambda_{1}^{2}+\lambda_{2}^{2}\right)
$$

They are the (complex) eigenvalues of an operator $\Lambda_{e}$, called the level shift operator associated to $e$ ( $\Lambda_{e}$ is related to the Lindblad generator). This operator acts on the eigenspace of $L_{\mathrm{S}}$ associated to the eigenvalue $e$ (a subspace of the qubit register Hilbert space; see equation (3.21) for the formal definition of $\Lambda_{e}$ ). It governs the lowest order shift of eigenvalues under perturbation. One can see by direct calculation that $\operatorname{Im} \delta_{e}^{(s)} \geq$ $0 .^{2}$

Discussion of (1.17). To lowest order in the perturbation, the group of reduced density matrix elements $\left[\bar{\rho}_{t}\right]_{\underline{\sigma}, \underline{\tau}}$ associated to a fixed $e=e(\underline{\sigma}, \underline{\tau})$ evolve in a coupled way, while groups of matrix elements associated to different $e$ evolve independently. The density matrix elements of a given group mix and evolve in time according to the weight functions $w$ and the exponentials $\mathrm{e}^{\mathrm{i} t \varepsilon_{e}^{(s)}}$. In the absence of interaction $\left(\lambda_{1}=\lambda_{2}=0\right)$ all

\footnotetext{
${ }^{1}$ Our method is applicable as well if this condition is not imposed. Work on this is in progress.

${ }^{2}$ This can also be inferred from general considerations [14]: If the imaginary part was negative, then the average of some observables would explode as time increases, contradicting the fact that the total dynamics, a group of automorphisms, cannot increase indefinitely the average of any observable.
} 
the $\varepsilon_{e}^{(s)}$ are real. As the interaction is switched on, the $\varepsilon_{e}^{(s)}$ typically migrate into the upper complex plane, but they may stay on the real line in certain cases. The matrix elements $\left[\bar{\rho}_{t}\right]_{\underline{\sigma}, \underline{\tau}}$ of a group $e$ approach their ergodic means (1.16) if and only if all the nonzero $\varepsilon_{e}^{(s)}$ have strictly positive imaginary part. In this case the convergence takes place on a time scale of the order $1 / \gamma_{e}$, where

$$
\gamma_{e}=\min \left\{\operatorname{Im} \varepsilon_{e}^{(s)}: s=1, \ldots, \nu(e) \text { s.t. } \varepsilon_{e}^{(s)} \neq 0\right\}
$$

is the decay rate of the group associated to $e$. If an $\varepsilon_{e}^{(s)}$ stays real then the matrix elements of the coresponding group oscillate in time. A sufficient condition for decay of the group associated to $e$ is $\gamma_{e}>0$, i.e. $\operatorname{Im} \delta_{e}^{(s)}>0$ for all $s$, and $\lambda_{1}, \lambda_{2}$ small.

Decoherence rates. We illustrate our results on decoherence rates for a qubit register with $J_{i j}=0$ (the general case is treated in Section 2.3). We consider generic magnetic fields defined as follows. For $n_{j} \in\{0, \pm 1, \pm 2\}, j=1, \ldots, N$, we have

$$
\sum_{j=1}^{N} B_{j} n_{j}=0 \quad \Longleftrightarrow \quad n_{j}=0 \forall j .
$$

Condition (1.21) is satisfied generically in the sense that only for very special choices of $B_{j}$ does it not hold (one such special choice is $B_{j}=$ constant). For instance, if the $B_{j}$ are chosen independent, and uniformly random from an interval $\left[B_{\min }, B_{\max }\right]$, then (1.21) is satisfied with probability one. We show in Theorem 2.3 that the decoherence rates $(1.20)$ are given by

$$
\gamma_{e}=\left\{\begin{array}{ll}
\lambda_{1}^{2} y_{1}(e)+\lambda_{2}^{2} y_{2}(e)+y_{12}(e), & e \neq 0 \\
\lambda_{2}^{2} y_{0}, & e=0
\end{array}\right\}+O\left(\lambda_{1}^{4}+\lambda_{2}^{4}\right) .
$$

Here, $y_{1}$ is a contributions coming from the energy conserving interaction, $y_{0}$ and $y_{2}$ are due to the spin flip interaction. The term $y_{12}$ is due to both interactions and is of $O\left(\lambda_{1}^{2}+\lambda_{2}^{2}\right)$. We give explicit expressions for $y_{0}, y_{1}, y_{2}$ and $y_{12}$ in equations (2.23), $(2.13),(2.15)$ and $(2.24)$.

- Properties of $y_{1}(e): y_{1}(e)$ vanishes if either $e$ is such that $e_{0}:=\sum_{j=1}^{n}\left(\sigma_{j}-\tau_{j}\right)=0$, or the infra-red behaviour of the coupling function $g_{1}$ is too regular (in three dimensions $g_{1} \propto|k|^{p}$ with $\left.p>-1 / 2\right)$. Otherwise $y_{1}(e)>0$. Moreover, $y_{1}(e)$ is proportional to the temperature $T$.

- Properties of $y_{2}(e): y_{2}(e)>0$ if $g_{2}\left(2 B_{j}, \Sigma\right) \neq 0$ for all $B_{j}$ (form factor $g_{2}(k)=$ $g_{2}(|k|, \Sigma)$ in spherical coordinates). For low temperatures $T, y_{2}(e) \propto T$, for high temperatures $y_{2}(e)$ approaches a constant.

- Properties of $y_{12}(e)$ : If either of $\lambda_{1}, \lambda_{2}$ or $e_{0}$ vanish, or if $g_{1}$ is infra-red regular as mentioned above, then $y_{12}(e)=0$. Otherwise $y_{12}(e)>0$, in which case $y_{12}(e)$ approaches constant values for both $T \rightarrow 0, \infty$. 
- Full decoherence: If $\gamma_{e}>0$ for all $e \neq 0$ then all off-diagonal matrix elements approach their limiting values exponentially fast. In this case we say that full decoherence occurs. It follows from the above points that we have full decoherence if $\lambda_{2} \neq 0$ and $g_{2}\left(2 B_{j}, \Sigma\right) \neq 0$ for all $j$, and provided $\lambda_{1}, \lambda_{2}$ are small enough (so that the remainder term in (1.22) is small). Note that if $\lambda_{2}=0$ then matrix elements associated to energy differences $e$ such that $e_{0}=0$ will not decay on the time scale given by the second order in the perturbation $\left(\lambda_{1}^{2}\right)$.

We point out that generically, $\mathrm{S}+\mathrm{R}$ will reach a joint equilibrium as $t \rightarrow \infty$, which means that the final reduced density matrix of $\mathrm{S}$ is its Gibbs state modulo a peturbation of the order of the interaction between $S$ and $R$, see [16]. Hence generically, the density matrix of $\mathrm{S}$ does not become diagonal in the energy basis as $t \rightarrow \infty$.

- Properties of $y_{0}: y_{0}$ depends on the energy exchange interaction only. This reflects the fact that for a purely energy conserving interaction, the populations are conserved $[16,17]$. If $g_{2}\left(2 B_{j}, \Sigma\right) \neq 0$ for all $j$, then $y_{0}>0$ (this is sometimes called the "Fermi Golden Rule Condition"). For small temperatures $T, y_{0} \propto T$, while $y_{0}$ approaches a finite limit as $T \rightarrow \infty$.

In terms of complexity analysis, it is important to discuss the dependence of $\gamma_{e}$ on the register size $N$.

- We see from (2.23) that $y_{0}$ is independent of $N$. This means that the thermalization time, or relaxation time of the diagonal matrix elements (corresponding to $e=0)$, is $O(1)$ in $N$.

- To determine the order of magnitude of the decay rates of the off-diagonal density matrix elements (corresponding to $e \neq 0$ ) relative to the register size $N$, we assume the magnetic field to have a certain distribution denoted by \langle\rangle . It follows from the explicit expressions for $y_{1}, y_{2}$ and $y_{12}$ (see (2.13), (2.15) and (2.24)) that

$$
\left\langle y_{1}\right\rangle=y_{1} \propto e_{0}^{2}, \quad\left\langle y_{2}\right\rangle=C_{B} \mathfrak{D}(\underline{\sigma}-\underline{\tau}), \quad \text { and } \quad\left\langle y_{12}\right\rangle=c_{B}\left(\lambda_{1}, \lambda_{2}\right) N_{0}(e)
$$

where $C_{B}$ and $c_{B}=c_{B}\left(\lambda_{1}, \lambda_{2}\right)$ are positive constants (independent of $N$ ), with $c_{B}\left(\lambda_{1}, \lambda_{2}\right)=O\left(\lambda_{1}^{2}+\lambda_{2}^{2}\right)$. Here, $N_{0}(e)$ is the number of indices $j$ such that $\sigma_{j}=\tau_{j}$ for each $(\underline{\sigma}, \underline{\tau})$ s.t. $e(\underline{\sigma}, \underline{\tau})=e$, and

$$
\mathfrak{D}(\underline{\sigma}-\underline{\tau}):=\sum_{j=1}^{N}\left|\sigma_{j}-\tau_{j}\right|
$$

is the Hamming distance between the spin configurations $\underline{\sigma}$ and $\underline{\tau}$ (which depends on $e$ only).

- Consider $e \neq 0$. It follows from (1.22)-(1.24) that for purely energy conserving interactions $\left(\lambda_{2}=0\right), \gamma_{e} \propto \lambda_{1}^{2} e_{0}^{2}=\lambda_{1}^{2}\left[\sum_{j=1}^{N}\left(\sigma_{j}-\tau_{j}\right)\right]^{2}$, which can be as large as $O\left(\lambda_{1}^{2} N^{2}\right)$. On the other hand, for purely energy exchanging interactions $\left(\lambda_{1}=0\right)$, we have $\gamma_{e} \propto \lambda_{2}^{2} \mathfrak{D}(\underline{\sigma}-\underline{\tau})$, which cannot exceed $O\left(\lambda_{2}^{2} N\right)$. If both interactions are 
acting, then we have the additional term $\left\langle y_{12}\right\rangle$, which is of order $O\left(\left(\lambda_{1}^{2}+\lambda_{2}^{2}\right) N\right)$. This shows the following:

The fastest decay rate of reduced off-diagonal density matrix elements due to the energy conserving interaction alone is of order $\lambda_{1}^{2} N^{2}$, while the fastest decay rate due to the energy exchange interaction alone is of the order $\lambda_{2}^{2} N$. Moreover, the decay of the diagonal matrix elements is of oder $\lambda_{1}^{2}$, i.e., independent of $N$.

- The same discussion is valid for the interacting register $\left(J_{i j} \neq 0\right)$, see Section 2.3.

Remarks. 1. For $\lambda_{2}=0$ the model can be solved explicitly [17], and one shows that the fastest decaying matrix elements have decay rate proportional to $\lambda_{1}^{2} N^{2}$. Furthermore, the model with a non-collective, energy-conserving interaction, where each qubit is coupled to an independent reservoir, can also be solved explicitly [17]. The fastest decay rate in this case is shown to be proportional to $\lambda_{1}^{2} N$.

2. As mentioned at the beginning of this section, we take the coupling constants $\lambda_{1}, \lambda_{2}$ so small that the resonances do not overlap (Condition (A1) in Section 2). Consequently $\lambda_{1}^{2} N^{2}$ and $\lambda_{2}^{2} N$ are bounded above by $\Delta=2 \min _{j=1, \ldots, N} B_{j}$ (see also Remark 4 after Condition (A1)) and thus the decay rates $\gamma_{e}$ do not increase indefinitely with increasing $N$ in the regime considered here. Rather, the $\gamma_{e}$ are attenuated by small coupling constants for large $N$. They are of the order $\gamma_{e} \sim \Delta$. We have shown that modulo an overall, common $(N$-dependent) prefactor, the decay rates originating from the energy conserving and exchanging interactions differ by a factor $N$.

In this paper we prove the results only for sufficiently high temperatures. The general case will be treated elsewhere.

3. The decay of off-diagonal matrix elements in the energy basis does not relate directly to measurements of entanglement, $[18,19]$. We plan on elucidating the interplay between entanglement and decay of matrix elements in a subsequent work.

Literature. Collective decoherence has been studied extensively in the literature. Among the many theoretical, numerical and experimental works we mention here only $[1,2,4,7,8,17,20]$, which are closest to the present work. We are not aware of any prior work giving explicit decoherence rates of a register for not explicitly solvable models, and without making master equation technique approximations.

\section{Results}

As mentioned in the introduction, we assume that

(A1) We have $C_{0}\left(\left|\lambda_{1}\right|+\left|\lambda_{2}\right|\right) N<\Delta$ for some constant $C_{0}$ (depending only on $g_{1}, g_{2}$, $J_{i j}$ and $\left.B_{j}\right)$. Here, $\Delta:=\min \left\{e-e^{\prime}: e, e^{\prime} \in \operatorname{spec}\left(L_{\mathrm{S}}\right), e \neq e^{\prime}\right\}$ is the gap in the spectrum of $L_{\mathrm{S}}$.

We implement a dynamical theory of resonances in a setting of spectral deformation (see Section 3.1). This leads to the following regularity requirement which we assume to be fulfilled throughout the paper. 
(A2) The function form factors $g_{1}, g_{2}$ in (1.4) satisfy the following condition. For $h=g_{1}$ or $h=g_{2}$,

$$
h_{\beta}(u, \sigma):=\sqrt{\frac{u}{1-\mathrm{e}^{-\beta u}}}|u|^{1 / 2} \begin{cases}h(u, \sigma) & \text { if } u \geq 0 \\ \mathrm{e}^{\mathrm{i} \phi} \bar{h}(-u, \sigma) & \text { if } u<0\end{cases}
$$

is such that $\omega \mapsto h_{\beta}(u+\omega, \sigma)$ has an analytic continuation, as a map $\mathbb{C} \rightarrow$ $L^{2}\left(\mathbb{R} \times S^{2}, \mathrm{~d} u \times \mathrm{d} \sigma\right)$, into $\{|\omega|<\tau\}$, for some $\tau>0$. Here, $\phi$ is an arbitrary fixed phase.

Remarks. 1. Typically, the gap $\Delta$ depends on $N$. We have $\left\|H_{\mathrm{S}}\right\|<C N^{2}$ and $\left\|L_{\mathrm{S}}\right\|<C N^{2}$, for some constant $C$. Therefore, if the $2^{N}\left(=\operatorname{dim} \mathcal{H}_{\mathrm{S}} \otimes \mathcal{H}_{\mathrm{S}}\right)$ eigenvalues of $L_{\mathrm{S}}$ are roughly simple and equally distributed, then the gap $\Delta$ is of the order $N^{2} 2^{-N}$. In this case, Condition (A1) implies that the coupling constants $\lambda_{1}$ and $\lambda_{2}$ have to be exponentially small in the size $N$ of the qubit register. However, the gap $\Delta$ tends to become larger as the multiplicities of the eigenvalues of $L_{\mathrm{S}}$ increase: $\Delta$ is the minimal distance between distinct eigenvalues of $L_{\mathrm{S}}$, spread over an interval of size $\left\|L_{\mathrm{S}}\right\|$. Due to the increase of multiplicities, the gap may become independent of $N$, as it happens in the following examples.

- For $H_{\mathrm{S}}=J \sum_{j=1}^{N} S_{j}^{z} S_{j+1}^{z}$ (nearest neighbour interaction; and say $S_{N+1}^{z} \equiv S_{1}^{z}$ ), we have $\operatorname{spec}\left(L_{\mathrm{S}}\right)=J\{-2 N,-2 N+1, \ldots, 2 N-1,2 N\}$. It follows that $\Delta=|J|$ is independent of $N$.

- For $H_{\mathrm{S}}=\sum_{j=1}^{N} B_{j} S_{j}^{z}$, the difference between two eigenvalues of $L_{\mathrm{S}}$ is given by $e-e^{\prime}=\sum_{j=1}^{N} B_{j}\left(n_{j}-n_{j}^{\prime}\right)$, where $n_{j}, n_{j}^{\prime} \in\{-2,0,2\}$. Hence (for $\left.B_{j}>0\right), \Delta=$ $2 \min _{j=1, \ldots, N} B_{j}$.

2. Examples of form factors satisfying (A2) are $g(k)=h_{1}(\sigma)|k|^{p} \mathrm{e}^{-|k|^{2}}$, where $p=-1 / 2+n, n=0,1,2, \ldots$, and $h_{1}(\sigma)=\mathrm{e}^{\mathrm{i} \phi} \bar{h}_{1}(\sigma)$. They include the physically most important cases, see also $[16,17]$. We point out that it is possible to weaken condition (A2) considerably, at the expense of a mathematically more involved treatment, as mentioned in [16]. The phase $\phi$ has been introduced, and its physical interpretation has been given, in [9].

\subsection{Effective dynamics of $\mathrm{S}$}

The main result of this section is Theorem 2.1, in which we describe the effective dynamics of $\mathrm{S}$ and identify the dominant part.

The evolution of reduced density matrix elements is governed by exponentials $\mathrm{e}^{\mathrm{i} t \varepsilon_{e}^{(s)}\left(\lambda_{1}, \lambda_{2}\right)}$, where $\varepsilon_{e}^{(s)}\left(\lambda_{1}, \lambda_{2}\right)$ are resonance energies, lying in the upper complex plane. The subindex $e$ is the eigenvalue of $L_{\mathrm{S}}$ which the resonance branches out of: $\varepsilon_{e}^{(s)}(0,0)=$ $e$, and the index $s=1, \ldots, \nu(e) \leq d(e)$ distinguishes different resonance energies associated to the same $e\left(d(e)\right.$ is the degeneracy of $e$ as an eigenvalue of $\left.L_{\mathrm{S}}\right)$. Using perturbation theory (we employ the Feshbach projection method (see Section 3.1 and $[5,16]))$, one obtains $(1.18)$.

Let $\left\{\eta_{e}^{(s, r)}\right\}_{r=1}^{R}$ and $\left\{\widetilde{\eta}_{e}^{(s, r)}\right\}_{r=1}^{R}$ be bases of the eigenspaces of the level shift operator 
$\Lambda_{e}$ and its adjoint $\Lambda_{e}^{*}$ (see (3.21) for the formal definition of $\Lambda_{e}$ ),

$$
\begin{aligned}
& \Lambda_{e} \eta_{e}^{(s, r)}=\delta_{e}^{(s)} \eta_{e}^{(s, r)}, \quad r=1, \ldots R, \\
& \Lambda_{e}^{*} \widetilde{\eta}_{e}^{(s, r)}=\overline{\delta_{e}^{(s)}} \widetilde{\eta}_{e}^{(s, r)}, \quad r=1, \ldots R,
\end{aligned}
$$

where $R=R(e, s)$ is the geometric multiplicity of the eigenvalue $\delta_{e}^{(s)}$ of $\Lambda_{e}$. We choose bases that are dual to each other, ${ }^{3}$ meaning that

$$
\left\langle\eta_{e}^{(s, r)}, \widetilde{\eta}_{e}^{\left(s, r^{\prime}\right)}\right\rangle=\delta_{r, r^{\prime}}
$$

We define the projection

$$
q_{e}^{(s)}=\sum_{r=1}^{R}\left|\eta_{e}^{(s, r)}\right\rangle\left\langle\widetilde{\eta}_{e}^{(s, r)}\right|,
$$

acting on the eigenspace of $L_{\mathrm{S}}$ associated to $e .^{4}$

Theorem 2.1 (Dynamics of matrix elements) Denote by $\beta$ the inverse temperature of $\mathrm{R}$. There is a $\lambda_{0}>0$ such that if $\max \left\{\left|\lambda_{1}\right|,\left|\lambda_{2}\right|\right\}<\lambda_{0} / \beta$ then the limit (1.16) exists for all $\underline{\sigma}, \underline{\tau}$, and we have for $t \geq 0$

$$
\begin{aligned}
& {\left[\bar{\rho}_{t}\right]_{\underline{\sigma}, \underline{\tau}}-\left\langle\left\langle\left[\bar{\rho}_{\infty}\right]_{\underline{\sigma}, \underline{\tau}}\right\rangle\right\rangle=} \\
& \sum_{\left\{e, s: \varepsilon_{e}^{(s)} \neq 0\right\}} \mathrm{e}^{\mathrm{i} t \varepsilon_{e}^{(s)}}\left[\sum_{\underline{\sigma}^{\prime}, \underline{\tau}^{\prime}}^{*}\left\langle\varphi_{\underline{\tau}^{\prime}, \underline{\sigma}^{\prime}}, q_{e}^{(s)} \varphi_{\underline{\tau}, \underline{\sigma}}\right\rangle\left[\bar{\rho}_{0}\right]_{\underline{\sigma}^{\prime}, \underline{\tau}^{\prime}}+R_{1}\right]+R_{2}(t) .
\end{aligned}
$$

The * in the last sum indicates that we only sum over spin configurations $\underline{\sigma}^{\prime}, \underline{\tau}^{\prime}$ such that $e\left(\underline{\sigma}^{\prime}, \underline{\tau}^{\prime}\right)=-e$. The remainders satisfy

$$
\left|R_{1}\right| \leq C N^{2}\left(\lambda_{1}^{2}+\lambda_{2}^{2}\right) \quad \text { and } \quad\left|R_{2}(t)\right| \leq C N^{2}\left(\lambda_{1}^{2}+\lambda_{2}^{2}\right) \mathrm{e}^{-\omega^{\prime} t},
$$

where $C$ is a constant, $N$ is the register size, and where $\omega^{\prime}$ satisfies $2 \max _{e, s}\left\{\operatorname{Im} \varepsilon_{e}^{(s)}\right\}<$ $\omega^{\prime}<\tau / 2$, with $\tau$ given in Condition (A2).

Remarks. 1. Since $q_{e}^{(s)}$ is a projection with range in the eigenspace associated to the eigenvalue $e$ of $L_{\mathrm{S}}$, we have $q_{e}^{(s)} \varphi_{\underline{\tau}, \underline{\sigma}}=0$ unless $e(\underline{\sigma}, \underline{\tau})=-e$ (see the scalar product in $(2.5))$.

2. The condition $\max \left\{\left|\lambda_{1}\right|,\left|\lambda_{2}\right|\right\}<\lambda_{0} / \beta$ stems from the particular complex deformation we choose in this work (translation). A mathematically more sophisticated treatment, involving a combination of spectral translation and dilation, and an iterative renormalization group analysis will yield the theorem for small $\lambda_{1}, \lambda_{2}$, but with a temperature independent upper bound (see also $[14,15]$ and remarks in [16]).

3. We mention again that in this work, we consider the regime of non-overlapping resonances, described by Condition (A1) at the beginning of Section (2). This means that $\lambda_{1}, \lambda_{2} \sim 1 / N$.

\footnotetext{
${ }^{3}$ This is always possible, see Proposition A.2 in Appendix A.

${ }^{4}$ This projection is the same for all choices of bases $\eta_{e}^{(s, r)}$ and $\widetilde{\eta}_{e}^{(s, r)}$ satisfying (2.3), as is easily verified using Proposition A.2 of Appendix A.
} 


\subsection{Non-interacting qubit register in magnetic field}

We consider the qubit register Hamiltonian (1.1) with $J_{i j}=0$ and $B_{j}>0$, with a coupling to the reservoir given by (1.4). In this section we determine the resonance eigenvectors $\eta_{e}^{(s, r)}, \widetilde{\eta}_{e}^{(s, r)}$ explicitly, as well as the resonance energies $\varepsilon_{e}^{(s)}$ to lowest order in the interaction, see Theorem 2.2. Those quantities are the key ingredients entering the dynamics which we describe in Theorem 2.4 below.

Let $\underline{\sigma}, \underline{\tau}$ be spin configurations of the form (1.8). Then

$$
\varphi_{\underline{\sigma}, \underline{\tau}}=\varphi_{\sigma_{1} \tau_{1}} \otimes \cdots \otimes \varphi_{\sigma_{N} \tau_{N}} \quad \text { with } \quad \varphi_{\sigma \tau}=\varphi_{\sigma} \otimes \varphi_{\tau} \in \mathbb{C}^{2} \otimes \mathbb{C}^{2}
$$

is an eigenvector of $L_{\mathrm{S}}$ with eigenvalue $e(\underline{\sigma}, \underline{\tau})=\sum_{j} B_{j}\left(\sigma_{j}-\tau_{j}\right)$. The genericness condition (1.21) implies that if $\varphi_{\underline{\sigma}, \underline{\tau}}$ and $\varphi_{\underline{\sigma}^{\prime}, \underline{\tau}^{\prime}}$ are eigenvectors associated to the same eigenvalue, then $\sigma_{j}-\tau_{j}=\sigma_{j}^{\prime}-\tau_{j}^{\prime}$ for all $j$. If $\sigma_{j}-\tau_{j}= \pm 2$ then $\sigma_{j}= \pm 1$ and $\tau_{j}=\mp 1$ are determined uniquely, while if $\sigma_{j}-\tau_{j}=0$, then there are two choices, $\sigma_{j}=\tau_{j}= \pm 1$. Consequently, an orthonormal basis of eigenvectors of $L_{\mathrm{S}}$ associated to a given eigenvalue $e$ can be constructed as follows. Take any one eigenvector $\varphi_{\underline{\sigma}, \underline{\tau}}$ associated to $e$ and adjoin all linearly independent vectors $\varphi_{\underline{\sigma}^{\prime}, \tau^{\prime}}$ with the property $\left\{\sigma_{j}-\tau_{j}=0\right\} \Leftrightarrow\left\{\sigma_{j}^{\prime}-\tau_{j}^{\prime}=0\right\}$. Thus, with each eigenvalue $e$ we associate the number

$$
N_{0}(e)=\left\{\text { number of indices } j \text { s.t. } \sigma_{j}=\tau_{j} \text { in any }(\underline{\sigma}, \underline{\tau}) \text { with } e(\underline{\sigma}, \underline{\tau})=e\right\} \text {, }
$$

and the degeneracy of the eigenvalue $e$ of $L_{\mathrm{S}}$ is $d(e)=2^{N_{0}(e)}$. To each eigenvalue $e$ of $L_{\mathrm{S}}$ there corresponds a unique sequence of $N_{0}(e)$ indices indicating the locations $j$ at which $\sigma_{j}=\tau_{j}$ for all $\underline{\sigma}, \underline{\tau}$ associated with $e$. In other words, given $e$ there is a unique sequence $\left\{\mu_{k}\right\}_{k=1}^{N_{0}(e)}$,

$$
1 \leq \mu_{1}<\mu_{2}<\cdots<\mu_{N_{0}(e)} \leq N
$$

having the property that any eigenvector $\varphi_{\underline{\sigma}, \underline{\tau}}$ associated to $e$ satisfies

$$
\sigma_{j}=\tau_{j} \quad \Longleftrightarrow \quad j \in\left\{\mu_{k}: k=1, \ldots, N_{0}(e)\right\} .
$$

Given an energy difference $e(1.13)$, and a sequence $\underline{\varrho}=\left(\varrho_{j}\right)_{j=1}^{N_{0}(e)}, \varrho_{j} \in\{+1,-1\}$, we set

$$
\delta_{e}^{(\underline{\varrho})}=\lambda_{1}^{2}\left[x_{1}(e)+\mathrm{i} y_{1}(e)\right]+\lambda_{2}^{2}\left[x_{2}(e)+\mathrm{i} y_{2}(e)\right]+\sum_{j=1}^{N_{0}(e)} z_{j}^{\varrho_{j}},
$$

where

$$
\begin{aligned}
x_{1}(e) & =-e_{0} \mathrm{P} \cdot \mathrm{V} \cdot\left\langle g_{1}, \omega^{-1} g_{1}\right\rangle \sum_{\left\{j: \sigma_{j}=\tau_{j}\right\}} \sigma_{j} \\
y_{1}(e) & =\frac{\pi e_{0}^{2}}{2 \beta} \gamma_{+}, \\
x_{2}(e) & =-\sum_{\left\{j: \sigma_{j} \neq \tau_{j}\right\}} \sigma_{j} \mathrm{P} \cdot \mathrm{V} \cdot \int_{\mathbb{R}} u^{2} \mathcal{G}_{2}(2 u) \operatorname{coth}(\beta|u|) \frac{1}{u-B_{j}} \mathrm{~d} u \\
y_{2}(e) & =2 \pi \sum_{\left\{j: \sigma_{j} \neq \tau_{j}\right\}} B_{j}^{2} \mathcal{G}_{2}\left(2 B_{j}\right) \operatorname{coth}\left(\beta B_{j}\right), \\
z_{j}^{ \pm} & =\frac{1}{2}\left[\mathrm{i} b_{j}\left(c_{j}+1\right) \pm \sqrt{-b_{j}^{2}\left(c_{j}+1\right)^{2}+4 a\left[a-\mathrm{i} b_{j}\left(c_{j}-1\right)\right]}\right],
\end{aligned}
$$


with

$$
a=-\lambda_{1}^{2} e_{0} \mathrm{P} . \mathrm{V} \cdot\left\langle g_{1}, \omega^{-1} g_{1}\right\rangle, \quad b_{j}=4 \pi \lambda_{2}^{2} \frac{B_{j}^{2} \mathcal{G}_{2}\left(2 B_{j}\right)}{\mathrm{e}^{2 \beta B_{j}}-1}, \quad c_{j}=e^{2 \beta B_{j}},
$$

and

$$
e_{0}=e_{0}(e)=\sum_{j=1}^{N}\left(\sigma_{j}-\tau_{j}\right), \quad \mathcal{G}_{k}(u)=\int_{S^{2}}\left|g_{k}(|u|, \Sigma)\right|^{2} \mathrm{~d} \Sigma, \quad \gamma_{+}=\lim _{u \rightarrow 0_{+}} u \mathcal{G}_{1}(u)
$$

The form factors $g_{1}, g_{2}$ (see (1.4)) are represented in spherical coordinates in (2.18) and P.V. stands for principal value. Note that $e_{0}$ is the same for all spin configurations $\underline{\sigma}, \underline{\tau}$ associated to the same energy $e=e(\underline{\sigma}, \underline{\tau})$. This follows from the genericness of the magnetic field, (1.21), see paragraph after (2.7). We show in Theorem 3.5 that $\operatorname{Im} z_{j}^{ \pm} \geq 0$. Let us define the vectors

$$
\begin{aligned}
& \eta_{e}^{(\underline{\underline{\rho}})}=\varphi_{\sigma_{1} \tau_{1}} \otimes \cdots \otimes \xi_{\mu_{1}}^{\varrho_{1}} \otimes \cdots \otimes \xi_{\mu_{N_{0}(e)}}^{\varrho_{N_{0}(e)}} \otimes \cdots \otimes \varphi_{\sigma_{N} \tau_{N}}, \\
& \widetilde{\eta}_{e}^{(\underline{\underline{\varrho}})}=\varphi_{\sigma_{1} \tau_{1}} \otimes \cdots \otimes \widetilde{\xi}_{\mu_{1}}^{\varrho_{1}} \otimes \cdots \otimes \widetilde{\xi}_{\mu_{N_{0}(e)}}^{\varrho_{N_{0}}(e)} \otimes \cdots \otimes \varphi_{\sigma_{N} \tau_{N}},
\end{aligned}
$$

where the $\varphi_{\sigma_{\mu_{j}} \tau_{\mu_{j}}}$ at positions $\mu_{j}, j=1, \ldots, N_{0}(e)$, are replaced by $\xi, \widetilde{\xi} \in \mathbb{C}^{2} \otimes \mathbb{C}^{2}$, given by

$$
\begin{aligned}
& \xi_{j}^{ \pm}=\varphi_{++}+\left[1+\mathrm{i} \frac{z_{j}^{ \pm}-a}{b_{j} c_{j}}\right] \varphi_{--} \\
& \widetilde{\xi}_{j}^{ \pm}=\varkappa_{j}^{ \pm}\left(\varphi_{++}+\left[1+\mathrm{i} \frac{z_{j}^{ \pm}-a}{b_{j} c_{j}}\right]^{*} \varphi_{--}\right),
\end{aligned}
$$

with normalization constant $\varkappa_{j}^{ \pm}=\left[1+b_{j}^{-2} c_{j}^{-1}\left\{\left(b_{j} c_{j}-\operatorname{Im} z_{j}^{ \pm}\right)^{2}+\left(a-\operatorname{Re} z_{j}^{ \pm}\right)^{2}\right\}\right]^{-1}$.

Theorem 2.2 (Resonance energies and states) Let e be an energy difference (1.13) and let $\Lambda_{e}$ be the associated level shift operator. The vectors $\eta_{e^{(\underline{\varrho})}}$ and $\widetilde{\eta}_{e^{\underline{e}}}$, (2.19) and (2.20), are bases of eigenvectors of $\Lambda_{e}$ and its adjoint $\Lambda_{e}^{*}$, respectively, which are dual to each other (see also (2.3)). The eigenvalues of $\Lambda_{e}$ and $\Lambda_{e}^{*}$ associated to $\eta_{e}^{(\underline{\underline{o}})}$ and $\widetilde{\eta}_{e}^{(\underline{\underline{\varrho}})}$ are given by $\delta_{e}^{(\underline{\varrho})}$, (2.11), and its complex conjugate, respectively. Furthermore, we have $\varepsilon_{e}^{(\underline{\underline{\varrho}})}=e+\delta_{e}^{(\underline{\underline{\varrho}})}+O\left(\lambda_{1}^{4}+\lambda_{2}^{4}\right)$.

Remark. The largest value of $N_{0}$ is $N$, which corresponds to $e=0$, so $d(0)=2^{N}$. Here, $\mu_{k}=k, k=1, \ldots, N$. The smallest value of $N_{0}$ is 0 , which corresponds to $e= \pm e_{\max }$, where $e_{\max }=2 \sum_{j} B_{j}$ is the largest eigenvalue of $L_{\mathrm{S}}$. Thus $e_{\max }$ is a simple eigenvalue of $L_{\mathrm{S}}$. Here, no two $\sigma_{j}, \tau_{j}$ are equal, so the sequence $\left\{\mu_{k}\right\}$ is "empty". We have $N_{0}(e)=N_{0}(-e)$, so $d(e)=d(-e)$ for all eigenvalues $e$.

The following result examines the resonance energies and shows expression (1.22) for the life times. 
Theorem 2.3 (Fermi Golden Rule Condition and decoherence rates) Assume that the so-called Fermi Golden Rule condition is satisfied:

$$
\lambda_{2}^{2} y_{0}:=4 \pi \lambda_{2}^{2} \min _{j=1, \ldots, N}\left\{B_{j}^{2} \mathcal{G}_{2}\left(2 B_{j}\right) \operatorname{coth}\left(\beta B_{j}\right)\right\}>0 .
$$

There is a $c>0$ s.t. if $\left|\lambda_{1}\right|,\left|\lambda_{2}\right|<c$, then the decoherence rates are given by (1.22), with

$$
y_{12}(e)=\sum_{\left\{j: \sigma_{j}=\tau_{j}\right\}} \min \left\{\operatorname{Im} z_{j}^{+}, \operatorname{Im} z_{j}^{-}\right\} .
$$

Remark. It is shown in Theorem 3.5 that $\operatorname{Im} z_{j}^{ \pm}>0$ provided $a b_{j} \neq 0$, and that if $a=0$, then $z_{j}^{+}=4 \pi \mathrm{i} \lambda_{2}^{2} B_{j}^{2} \mathcal{G}_{2}\left(2 G_{j}\right) \operatorname{coth}\left(\beta B_{j}\right), z_{j}^{-}=0$ and if $b_{j}=0$ then $z_{j}^{ \pm}= \pm a$. If $a b_{j}=0$ for all $j$ then $y_{12}(e)=0$.

Let us illustrate how Theorems 2.1, 2.2 and 2.3 combine to give the detailed dynamics of the register. Suppose that $\lambda_{2} \neq 0$. It is clear that for generic values of the magnetic field, all $\delta_{e}^{(\underline{\varrho})}$ are different for different $\underline{\varrho}$ (see (2.11)). Thus all resonance energies $\varepsilon_{e}^{(\underline{\underline{\varrho}})}=e+\delta_{e}^{\underline{(\underline{\varrho})}}+O\left(\lambda_{1}^{2}+\lambda_{2}^{2}\right)$ are simple, for small enough $\lambda_{1}, \lambda_{2}$. In this situation we obtain the following result:

Theorem 2.4 (Dominant dynamics) Suppose $\lambda_{2} \neq 0$ and suppose that the magnetic field is generic so that all $\delta_{e}^{(\underline{\underline{e}})}$, (2.11), are distinct. There is a constant $c$ s.t. if $\left|\lambda_{1}\right|+\left|\lambda_{2}\right|<c$, then we have for all $\underline{\sigma}, \underline{\tau}$

$$
\begin{aligned}
& {\left[\bar{\rho}_{t}\right]_{\underline{\sigma}, \underline{\tau}}-\left\langle\left\langle\left[\bar{\rho}_{\infty}\right]_{\underline{\sigma}, \underline{\tau}}\right\rangle\right\rangle=} \\
& \sum_{\left\{\underline{\varrho}: \varepsilon_{e}^{(\underline{\underline{\varrho}})} \neq 0\right\}} \mathrm{e}^{\mathrm{i} t \varepsilon_{e}^{(\underline{\underline{\varrho}})}}\left[\sum_{\underline{\sigma}^{\prime}, \underline{\tau}^{\prime}}^{*} w_{\underline{\sigma}, \underline{\underline{\tau}} ; \underline{\sigma}^{\prime}, \underline{\tau}^{\prime}}^{(e, \underline{\rho})}\left[\bar{\rho}_{0}\right]_{\underline{\sigma}^{\prime}, \underline{\tau}^{\prime}}+R_{1}\right]+R_{2},
\end{aligned}
$$

where the * means that we sum only over spin configurations s.t. $e\left(\underline{\sigma}^{\prime}, \underline{\tau}^{\prime}\right)=-e$, where $\varepsilon_{e}^{(\underline{\underline{\varrho}})}=e+\delta_{e}^{(\underline{\underline{\varrho}})}+O\left(\lambda_{1}^{4}+\lambda_{2}^{4}\right)$, the remainder terms $R_{1}, R_{2}$ satisfy (2.6), and where

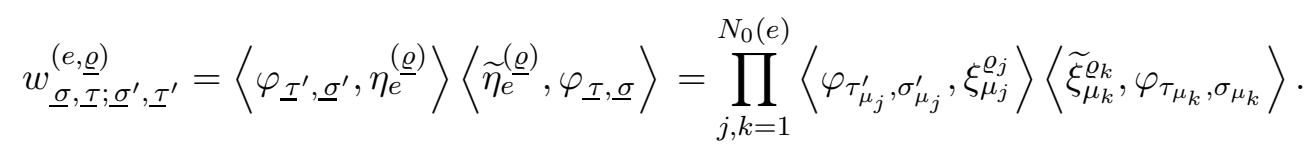

\subsection{Interacting qubit register in magnetic field}

In this section we consider the Hamiltonian $H_{\mathrm{S}},(1.1)$, with generic parameters $J_{i j}$ and $B_{j}$. Energy differences of $H_{\mathrm{S}}$ are

$$
e(\underline{\sigma}, \underline{\tau})=E(\underline{\sigma})-E(\underline{\tau})=\sum_{i, j=1}^{N} J_{i j}\left(\sigma_{i} \sigma_{j}-\tau_{i} \tau_{j}\right)+\sum_{j=1}^{N} B_{j}\left(\sigma_{j}-\tau_{j}\right) .
$$


The condition $e(\underline{\sigma}, \underline{\tau})=e\left(\underline{\sigma}^{\prime}, \underline{\tau}^{\prime}\right)$ is equivalent to

$$
\sum_{i, j=1}^{N} J_{i j} m_{i j}+\sum_{j=1}^{N} B_{j} n_{j}=0
$$

where $m_{i j}=\sigma_{i} \sigma_{j}-\sigma_{i}^{\prime} \sigma_{j}^{\prime}-\left[\tau_{i} \tau_{j}-\tau_{i}^{\prime} \tau_{j}^{\prime}\right]$ and $n_{j}=\sigma_{j}-\sigma_{j}^{\prime}-\left[\tau_{j}-\tau_{j}^{\prime}\right]$. For generic values of $J_{i j}$ and $B_{j}$, the only solution of $(2.27)$ is $m_{i j}=0, n_{j}=0$ for all $i, j=1, \ldots, N .^{5}$

Theorem 2.5 Let e be a nonzero eigenvalue of $L_{\mathrm{S}}$. The resonance energies associated to $e$ are $\varepsilon_{e}(\underline{\sigma}, \underline{\tau})=e+\delta_{e}(\underline{\sigma}, \underline{\tau})+O\left(\lambda_{1}^{4}+\lambda_{2}^{4}\right)$, where $(\underline{\sigma}, \underline{\tau})$ varies over all spin configurations s.t. $e(\underline{\sigma}, \underline{\tau})=e$, and where (omitting $(\underline{\sigma}, \underline{\tau})$ in the notation)

$$
\delta_{e}=\lambda_{1}^{2}\left[x_{1}+\mathrm{i} y_{1}\right]+\lambda_{2}^{2}\left[x_{2}+\mathrm{i} y_{2}\right]
$$

with $x_{1}(e)$ and $y_{1}(e)$ given in (2.12) and (2.13), and

$$
\begin{aligned}
& x_{2}=-\frac{1}{2} \sum_{k=1}^{N} \mathrm{P} \cdot \mathrm{V} \cdot \int_{\mathbb{R} \times S^{2}} u^{2}\left|g_{2}(u, \Sigma)\right|^{2}\left[\frac{\left|1-\mathrm{e}^{\beta u}\right|^{-1}}{u+v_{k}}+\frac{\left|1-\mathrm{e}^{-\beta u}\right|^{-1}}{u+v_{k}^{\prime}}\right] \\
& y_{2}=\frac{\pi}{2} \sum_{k=1}^{N}\left[\frac{v_{k}^{2} \mathcal{G}_{2}\left(v_{k}\right)}{\left|1-\mathrm{e}^{\beta v_{k}}\right|}+\frac{\left(v_{k}^{\prime}\right)^{2} \mathcal{G}_{2}\left(v_{k}^{\prime}\right)}{\mid 1-\mathrm{e}^{-\beta v_{k}^{\prime} \mid}}\right] .
\end{aligned}
$$

Here, $e_{0}$ is given in (2.18) and

$$
v_{k}=-2 \sigma_{k}\left[\sum_{j=1}^{N}\left(J_{j k}+J_{k j}\right) \sigma_{j}+B_{k}\right], \quad v_{k}^{\prime}=2 \tau_{k}\left[\sum_{j=1}^{N}\left(J_{j k}+J_{k j}\right) \tau_{j}+B_{k}\right] .
$$

The resonance eigenvectors associated to the resonance energy $\varepsilon_{e}(\underline{\sigma}, \underline{\tau})$ are $\eta_{e(\underline{\sigma}, \underline{\tau})}=$ $\varphi_{\underline{\sigma}, \underline{\tau}}=\widetilde{\eta}_{e(\underline{\sigma}, \underline{\tau})}(\operatorname{see}(2.1),(2.2))$.

This result shows that the decoherence rates induced by the energy conserving interaction are again maximally $O\left(\lambda_{1}^{2} N^{2}\right)$, as in the case of the non-interacting register $\left(J_{i j}=0\right)$. However, the decoherence rates induced by the exchange interaction have a complicated dependence on $N: y_{2}$ is a sum of $N$ terms each one depending on $N$, the coupling parameters $J_{i j}$ and the magnetic field $B_{j}$.

Remark. One can proceed as for the non-interacting register (Section 2.2) to analyze the resonances bifurcating out of the origin (determined to lowest nontrivial order by the spectrum of the level shift operator $\Lambda_{0}$ ). One finds that $\Lambda_{0}$ has a simple eigenvalue at zero, and that the imaginary part of the smallest (nonzero) resonance is given by

$$
\gamma_{0}=4 \pi \lambda_{2}^{2} \min _{j=1, \ldots, N}\left\{\frac{C_{j,+}^{2} \mathcal{G}_{2}\left(2 C_{j,+}\right)}{\mid 1-\mathrm{e}^{-2 \beta C_{j,+} \mid}}+\frac{C_{j,-}^{2} \mathcal{G}_{2}\left(2 C_{j,-}\right)}{\mid 1-\mathrm{e}^{-2 \beta C_{j,-} \mid}}\right\},
$$

where

$$
C_{j, \pm}=\sum_{k=1}^{N}\left(J_{j k}+J_{k j}\right) \pm B_{j} .
$$

\footnotetext{
${ }^{5}$ Indeed, to solve (2.27) with some $m_{i j}$ or $n_{j}$ nonzero means to introduce some correlations among the parameters $J_{i j}$ and $B_{j}$. Note that in particular, $J_{i j}=J$ and $B_{j}=B$ is not a generic choice of parameters.
} 


\section{Proofs}

\subsection{Proof of Theorem 2.1}

In Theorem 3.2, we first obtain a suitable expression for the average $\langle A\rangle_{t}$ of an observable $A \in B\left(\mathcal{H}_{\mathrm{S}}\right)$. This result is based on the dynamical resonance theory developed in $[16]$, see also $[10,11]$, which we outline below. In a second step, we carry out a refined analysis of the resonance theory to obtain Theorem 3.3. The combination of Theorems 3.2 and 3.3 shows Theorem 2.1 .

Let $A \in B\left(\mathcal{H}_{\mathrm{S}}\right)$. We have

$$
\begin{aligned}
\langle A\rangle_{t} & =\operatorname{Tr}_{\mathrm{S}}\left[\bar{\rho}_{t} A\right]=\operatorname{Tr}_{\mathrm{S}+\mathrm{R}}\left[\rho_{t} A \otimes \mathbb{1}_{\mathrm{R}}\right] \\
& =\left\langle\psi_{0}, \mathrm{e}^{\mathrm{i} t L_{\lambda_{1}, \lambda_{2}}}\left[A \otimes \mathbb{1}_{\mathrm{S}} \otimes \mathbb{1}_{\mathrm{R}}\right] \mathrm{e}^{-\mathrm{i} t L_{\lambda_{1}, \lambda_{2}}} \psi_{0}\right\rangle .
\end{aligned}
$$

In the last step, we pass to the representation Hilbert space of the system (the GNS Hilbert space), where the initial density matrix is represented by the vector $\psi_{0}$ (in particular, the Hilbert space of the small system becomes $\mathcal{H}_{\mathrm{S}} \otimes \mathcal{H}_{\mathrm{S}}$ ).

The dynamics of an observable $A$ is implemented by the group of automorphisms $A \mapsto \mathrm{e}^{\mathrm{i} t L_{\lambda_{1}, \lambda_{2}}} A \mathrm{e}^{-\mathrm{i} t L_{\lambda_{1}, \lambda_{2}}}$. The self-adjoint generator $L_{\lambda_{1}, \lambda_{2}}$ is called the Liouville operator. It is of the form $L_{\lambda_{1}, \lambda_{2}}=L_{0}+\lambda_{1} W_{1}+\lambda_{2} W_{2}$, where $L_{0}=L_{\mathrm{S}}+L_{\mathrm{R}}$ represents the uncoupled Liouville operator, and $\lambda_{1} W_{1}+\lambda_{2} W_{2}$ is the interaction (represented in the GNS Hilbert space).

We take the initial state to be represented by the product vector $\psi_{0}=\psi_{\mathrm{S}, 0} \otimes \psi_{\mathrm{R}}$ (the product form of the initial state is actually not necessary for our method to work, see [16]). Here, $\psi_{\mathrm{S}, 0}$ is an arbitrary initial state of $\mathrm{S}$, and $\psi_{\mathrm{R}}$ is the equilibrium state of $\mathrm{R}$ at a fixed inverse temperature $0<\beta<\infty$. We denote by $\psi_{\mathrm{S}, \infty}$ the trace state of $\mathrm{S},\left\langle\psi_{\mathrm{S}, \infty},\left(A_{\mathrm{S}} \otimes \mathbb{1}_{\mathrm{S}}\right) \psi_{\mathrm{S}, \infty}\right\rangle=2^{-N} \operatorname{Tr}\left(A_{\mathrm{S}}\right)$. We introduce the reference vector

$$
\psi_{\mathrm{ref}}=\psi_{\mathrm{S}, \infty} \otimes \psi_{\mathrm{R}} .
$$

The trace state has the separating property: given any state $\psi_{\mathrm{S}, 0}$ there is a (unique) operator $B \in \mathfrak{M}_{\mathrm{S}}$, satisfying $\psi_{\mathrm{S}, 0}=\left(\mathbb{1}_{\mathrm{S}} \otimes B\right) \psi_{\mathrm{S}, \infty}$. We write $B^{\prime}:=\mathbb{1}_{\mathrm{S}} \otimes B$ and note that $B^{\prime}$ commutes with all observables, so that we obtain from (3.1)

$$
\langle A\rangle_{t}=\left\langle\psi_{0}, B^{\prime} \mathrm{e}^{\mathrm{i} t L_{\lambda_{1}, \lambda_{2}}}\left[A \otimes \mathbb{1}_{\mathrm{S}} \otimes \mathbb{1}_{\mathrm{R}}\right] \mathrm{e}^{-\mathrm{i} t L_{\lambda_{1}, \lambda_{2}}} \psi_{\text {ref }}\right\rangle .
$$

We now borrow a trick from the analysis of open systems far from equilibrium: one can find a (non-self-adjoint) generator $K_{\lambda_{1}, \lambda_{2}}$ s.t.

$$
\begin{aligned}
& \mathrm{e}^{\mathrm{i} t L_{\lambda_{1}, \lambda_{2}} A \mathrm{e}^{-\mathrm{i} t L_{\lambda_{1}, \lambda_{2}}}}=\mathrm{e}^{\mathrm{i} t K_{\lambda_{1}, \lambda_{2}} A \mathrm{e}^{-\mathrm{i} t K_{\lambda_{1}, \lambda_{2}}}} \text { for all observables } A, t \geq 0, \text { and } \\
& K_{\lambda_{1}, \lambda_{2}} \psi_{\text {ref }}=0 .
\end{aligned}
$$

There is a standard way of constructing $K_{\lambda_{1}, \lambda_{2}}$ given $L_{\lambda_{1}, \lambda_{2}}$ and the reference vector $\psi_{\text {ref. }} . K_{\lambda_{1}, \lambda_{2}}$ is of the form $K_{\lambda_{1}, \lambda_{2}}=L_{0}+\lambda_{1} I_{1}+\lambda_{2} I_{2}$, where the interaction terms appearing in the expression for $L_{\lambda_{1}, \lambda_{2}}$ undergo a modification $\lambda_{1} W_{1}+\lambda_{2} W_{2} \rightarrow \lambda_{1} I_{1}+$ $\lambda_{2} I_{2}$, c.f. [16]. Formally, we may replace the propagators in (3.3) by those involving 
$K_{\lambda_{1}, \lambda_{2}}$, and use that $\mathrm{e}^{-\mathrm{i} t K_{\lambda_{1}, \lambda_{2}}} \psi_{\text {ref }}=\psi_{\text {ref }}$. This procedure has been carried out in a rigorous manner in [16], yielding the following resolvent representation

$$
\langle A\rangle_{t}=-\frac{1}{2 \pi \mathrm{i}} \int_{\mathbb{R}-\mathrm{i}}\left\langle\psi_{0}, B^{\prime}\left(K_{\lambda_{1}, \lambda_{2}}(\omega)-z\right)^{-1}\left[A \otimes \mathbb{1}_{\mathrm{S}} \otimes \mathbb{1}_{\mathrm{R}}\right] \psi_{\text {ref }}\right\rangle \mathrm{e}^{\mathrm{i} t z} \mathrm{~d} z
$$

where $K_{\lambda_{1}, \lambda_{2}}(\omega)=L_{0}(\omega)+\lambda_{1} I_{1}(\omega)+\lambda_{2} I_{2}(\omega), I_{1,2}$ are representing the interactions, and $\omega \mapsto K_{\lambda_{1}, \lambda_{2}}(\omega)$ is a spectral deformation (translation) of $K_{\lambda_{1}, \lambda_{2}}$. Relation (3.4) holds for $0<\operatorname{Im} \omega<\tau$ (see condition (A2) in Section 2); the integrand is analytic in that domain, and continuous as $\operatorname{Im} \omega \downarrow 0$. For $\omega \in \mathbb{R}$, the integrand is independent of $\omega$ and so it is constant for all $\omega$ in the domain of analyticity.

The spectral deformation is constructed as follows. There is a deformation transformation $U(\omega)=\mathrm{e}^{-\mathrm{i} \omega D}$, where $D$ is the (explicit) self-adjoint generator of translations [16] transforming the operator $K_{\lambda_{1}, \lambda_{2}}$ as

$$
K_{\lambda_{1}, \lambda_{2}}(\omega)=U(\omega) K_{\lambda_{1}, \lambda_{2}} U(\omega)^{-1}=L_{0}+\omega N+\lambda_{1} I_{1}(\omega)+\lambda_{2} I_{2}(\omega) .
$$

Here, $N$ is the total number operator of $\mathcal{H}_{\mathrm{R}}$, having spectrum $\mathbb{N} \cup\{0\}$, where 0 is a simple eigenvalue (vacuum eigenvector $\psi_{\mathrm{R}}$ ). For real values of $\omega, U(\omega)$ is a group of unitaries. The spectrum of $K_{\lambda_{1}, \lambda_{2}}(\omega)$ depends on $\operatorname{Im} \omega$ and moves according to the value of $\operatorname{Im} \omega$, whence the name "spectral deformation". Even though $U(\omega)$ becomes unbounded for complex $\omega$, the r.h.s. of (3.5) is a well defined closed operator on a dense domain, analytic in $\omega$ at zero. Analyticity is used in the derivation of (3.4) and this is where the analyticity condition (A2) of Section 2 comes into play.

The point of the spectral deformation is that the (important part of the) spectrum of $K_{\lambda_{1}, \lambda_{2}}(\omega)$ is much easier to analyze than that of $K_{\lambda_{1}, \lambda_{2}}$, because the deformation uncovers the resonances of $K_{\lambda_{1}, \lambda_{2}}$. We have

$$
\operatorname{spec}\left(K_{0}(\omega)\right)=\left\{E_{i}-E_{j}\right\}_{i, j=1, \ldots, N} \bigcup_{n \geq 1}\{\omega n+\mathbb{R}\},
$$

because $K_{0}(\omega)=L_{0}+\omega N, L_{0}$ and $N$ commute, and the eigenvectors of $L_{0}=L_{\mathrm{S}}+L_{\mathrm{R}}$ are $\varphi_{i} \otimes \varphi_{j} \otimes \psi_{\mathrm{R}}$. The continuous spectrum of $K_{0}$ is bounded away from the isolated eigenvalues by a gap of size $\operatorname{Im} \omega$. The operator $\lambda_{1} I_{1}(\omega)+\lambda_{2} I_{2}(\omega)$ is infinitesimally small with respect to the number operator $N$, so for values of the coupling parameters $\lambda_{1,2}$ small compared to $\operatorname{Im} \omega$, we can follow the displacements of the eigenvalues by using analytic perturbation theory. The following is an easy result (see e.g. [14]).

Theorem 3.1 Fix $\omega^{\prime}$ s.t. $0<\omega^{\prime}<\tau$ (where $\tau$ is as in Condition (A2) of Section 2). There is a constant $c_{0}>0$ s.t. if $\max \left\{\left|\lambda_{1}\right|,\left|\lambda_{2}\right|\right\} \leq c_{0} / \beta$ ( $\beta$ is the inverse temperature) then, for all $\omega$ with $\omega^{\prime}<\omega<\tau$, the spectrum of $K_{\lambda_{1}, \lambda_{2}}(\omega)$ in the complex half-plane $\left\{\operatorname{Im} z<\omega^{\prime} / 2\right\}$ is independent of $\omega$ and consists purely of the distinct eigenvalues

$$
\left\{\varepsilon_{e}^{(s)}: e \in \operatorname{spec}\left(L_{\mathrm{S}}\right), s=1, \ldots, \nu(e)\right\},
$$

where $1 \leq \nu(e) \leq \operatorname{mult}(e)$ counts the splitting of the eigenvalue e. Moreover, we have $\varepsilon_{e}^{(s)}\left(\lambda_{1}, \lambda_{2}\right) \rightarrow e$ as $\lambda_{1}, \lambda_{2} \rightarrow 0$, for all $e, s$, and furthermore, $\operatorname{Im} \varepsilon_{e}^{(s)} \geq 0$. Also, the continuous spectrum of $K_{\lambda_{1}, \lambda_{2}}(\omega)$ lies in the region $\left\{\operatorname{Im} z \geq 3 \omega^{\prime} / 4\right\}$. 
Next we separate the contributions to the path integral in (3.4) coming from the singularities at the resonance energies and from the continuous spectrum. We deform the path of integration $z=\mathbb{R}-\mathrm{i}$ into the line $z=\mathbb{R}+\mathrm{i} \omega^{\prime} / 2$, thereby picking up the residues of poles of the integrand at $\varepsilon_{e}^{(s)}$ (all $\left.e, s\right)$. Let $\mathcal{C}_{e}^{(s)}$ be a small circle around $\varepsilon_{e}^{(s)}$, not enclosing or touching any other spectrum of $K_{\lambda_{1}, \lambda_{2}}(\omega)$. We introduce the (generally non-orthogonal) Riesz spectral projections

$$
Q_{e}^{(s)}=Q_{e}^{(s)}\left(\omega, \lambda_{1}, \lambda_{2}\right)=-\frac{1}{2 \pi \mathrm{i}} \int_{\mathcal{C}_{e}^{(s)}}\left(K_{\lambda_{1}, \lambda_{2}}(\omega)-z\right)^{-1} \mathrm{~d} z
$$

It follows from (3.4) that

$$
\langle A\rangle_{t}=\sum_{e} \sum_{s=1}^{\nu(e)} \mathrm{e}^{\mathrm{i} t \varepsilon_{e}^{(s)}}\left\langle\psi_{0}, B^{\prime} Q_{e}^{(s)}\left[A \otimes \mathbb{1}_{\mathrm{S}} \otimes \mathbb{1}_{\mathrm{R}}\right] \psi_{\mathrm{ref}}\right\rangle+R_{2},
$$

where the remainder term $R_{2}$ comes from the contour integral enclosing the continuous spectrum and satisfies

$$
\left|R_{2}\right| \leq C N^{2}\left(\lambda_{1}^{2}+\lambda_{2}^{2}\right) \mathrm{e}^{-\omega^{\prime} t / 2},
$$

for some constant $C$ not depending on the dimension $N$ of $\mathcal{H}_{\mathrm{S}}$, nor on $\lambda_{1}, \lambda_{2}$. Note that $R_{2}$ decays faster in time than each term in the main part. The estimate (3.8) is a direct consequence of Proposition 4.2 in [16] (see in particular equation (D.5) in the proof of this proposition).

The ergodic mean time limits of $R_{2}$ and all terms in (3.7) with $\varepsilon_{e}^{(s)} \neq 0$ vanish, so

$$
\langle\langle A\rangle\rangle_{\infty}:=\lim _{T \rightarrow \infty} \frac{1}{T} \int_{0}^{T}\langle A\rangle_{t} \mathrm{~d} t=\sum_{\left\{e, s: \varepsilon_{e}^{(s)}=0\right\}}\left\langle\psi_{0}, B^{\prime} Q_{0}^{(s)}\left[A \otimes \mathbb{1}_{\mathrm{R}} \otimes \mathbb{1}_{\mathrm{R}}\right] \psi_{\mathrm{ref}}\right\rangle .
$$

Combining the latter expression with (3.7) gives the following result.

Theorem 3.2 For $\max \left\{\left|\lambda_{1}\right|,\left|\lambda_{2}\right|\right\} \leq c_{0} / \beta$ (see Theorem 3.1), we have for $t \geq 0$ and $A \in \mathcal{B}\left(\mathcal{H}_{\mathrm{S}}\right)$

$$
\langle A\rangle_{t}-\langle\langle A\rangle\rangle_{\infty}=\sum_{\left\{e, s: \varepsilon_{e}^{(s)} \neq 0\right\}} \mathrm{e}^{\mathrm{i} t \varepsilon_{e}^{(s)}}\left\langle\psi_{0}, B^{\prime} Q_{e}^{(s)}\left[A \otimes \mathbb{1}_{\mathrm{S}} \otimes \mathbb{1}_{\mathrm{R}}\right] \psi_{\text {ref }}\right\rangle+R_{2},
$$

where $R_{2}$ satisfies (3.8).

Choosing $A=\left|\varphi_{\underline{\tau}}\right\rangle\left\langle\varphi_{\underline{\sigma}}\right|$ gives $\langle A\rangle_{t}=\left[\bar{\rho}_{t}\right]_{\underline{\sigma}, \underline{\tau}}$.

Theorem 3.3 Take $\max \left\{\left|\lambda_{1}\right|,\left|\lambda_{2}\right|\right\} \leq c_{0} / \beta$ (see Theorem 3.1), let e be any eigenvalue of $L_{\mathrm{S}}$ and let $\underline{\sigma}, \underline{\mathcal{\tau}}$ be spin configurations. We have for $t \geq 0$

$$
\begin{aligned}
& \left\langle\psi_{0}, B^{\prime} Q_{e}^{(s)}\left[\left|\varphi_{\underline{\tau}}\right\rangle\left\langle\varphi_{\underline{\sigma}}\right| \otimes \mathbb{1}_{\mathrm{S}} \otimes \mathbb{1}_{\mathrm{R}}\right] \psi_{\mathrm{ref}}\right\rangle= \\
& \sum_{\left\{\underline{\sigma}^{\prime}, \underline{\tau}^{\prime}: e\left(\underline{\sigma}^{\prime}, \underline{\tau}^{\prime}\right)=-e\right\}}\left\langle\varphi_{\underline{\tau}^{\prime}, \underline{\sigma}^{\prime}}, q_{e}^{(s)} \varphi_{\underline{\tau}, \underline{\sigma}}\right\rangle\left[\bar{\rho}_{0}\right]_{\underline{\sigma}^{\prime}, \underline{\tau}^{\prime}}+O\left(N^{2}\left(\lambda_{1}^{2}+\lambda_{2}^{2}\right)\right),
\end{aligned}
$$

where $q_{e}^{(s)}$ is defined in (2.4), and $N=\operatorname{dim} \mathcal{H}_{\mathrm{S}}$ is the register size. 
We point out that $q_{e}^{(s)} \varphi_{\underline{\tau}, \underline{\sigma}}$ vanishes unless $e(\underline{\tau}, \underline{\sigma})=e$. Theorem 2.1 now follows directly from Theorems 3.2 and 3.3.

Proof of Theorem 3.3. Let $R$ be the rank of $Q_{e}^{(s)}$ and $\left[Q_{e}^{(s)}\right]^{*}$, and let $\left\{\chi_{e}^{(s, r)}\right\}_{r=1}^{R}$ and $\left\{\widetilde{\chi}_{e}^{(s, r)}\right\}_{r=1}^{R}$ be bases of the ranges of those projections which are dual to each other, so that, by Proposition A.1,

$$
Q_{e}^{(s)}=\sum_{r=1}^{R}\left|\chi_{e}^{(s, r)}\right\rangle\left\langle\widetilde{\chi}_{e}^{(s, r)}\right|
$$

We have

$$
K_{\lambda_{1}, \lambda_{2}}(\omega) \chi_{e}^{(s, r)}=\varepsilon_{e}^{(s)} \chi_{e}^{(s, r)} \text { and }\left[K_{\lambda_{1}, \lambda_{2}}(\omega)\right]^{*} \widetilde{\chi}_{e}^{(s, r)}=\overline{\varepsilon_{e}^{(s)}} \widetilde{\chi}_{e}^{(s, r)} .
$$

The following isospectrality result is inferred from the Feshbach method, see [5], Section II and also [16]. We denote by $P_{e}$ the spectral projection of $K_{0}$ associated to the eigenvalue $e$, and we set $\bar{P}_{e}=1-P_{e}$.

Lemma 3.4 (Feshbach map) Let $\chi$ be an eigenvector of $K_{\lambda_{1}, \lambda_{2}}(\omega)$ with eigenvalue $\varepsilon$ (bifurcating out of $e$ ). Then $\xi=P_{e} \chi$ is an eigenvector of the operator

$$
P_{e}\left[e-I(\omega) \bar{P}_{e}\left(\bar{K}_{\lambda_{1}, \lambda_{2}}(\omega)-\varepsilon\right)^{-1} \bar{P}_{e} I(\omega)\right] P_{e}
$$

with eigenvalue $\varepsilon$. Conversely, if $\xi \in \operatorname{Ran} P_{e}$ is an eigenvector of the operator (3.12) with eigenvalue $\varepsilon$, then

$$
\chi=\left[\mathbb{1}-\bar{P}_{e}\left(\bar{K}_{\lambda_{1}, \lambda_{2}}(\omega)-\varepsilon\right)^{-1} \bar{P}_{e} I(\omega)\right] P_{e} \xi
$$

is an eigenvector of $K_{\lambda_{1}, \lambda_{2}}(\omega)$ with eigenvalue $\varepsilon$. Moreover, if $\chi$ is an eigenvector as above, then $\xi=P_{e} \chi \neq 0$ and conversely, if $\xi$ is an eigenvector as above, then $\chi$ given in (3.13) is nonzero. In particular, the geometric multiplicity of $\varepsilon$ as an eigenvalue of $K_{\lambda_{1}, \lambda_{2}}(\omega)$ is the same as that of $\varepsilon$ as an eigenvalue of (3.12).

Expanding the resolvent in $(3.12)$ around $\left(\bar{L}_{0}(\omega)-e\right)^{-1}$ we obtain for $\xi=\xi_{e}^{(s, r)}$

$$
\xi_{e}^{(s, r)}=\left[\eta_{e}^{(s, r)}+O\left(N^{2}\left(\lambda_{1}^{2}+\lambda_{2}^{2}\right)\right)\right] \otimes \psi_{\mathrm{R}},
$$

where $\eta_{e}^{(s, r)}$ satisfies (2.1), with the level shift operator $\Lambda_{e}$ defined in (3.21), and where $N=\operatorname{dim} \mathcal{H}_{\mathrm{S}}$. We expand the resolvent in (3.13) around $\left(\bar{L}_{0}(\omega)-e\right)^{-1}$ and use (3.14) to obtain

$$
\chi_{e}^{(s, r)}=\left[\mathbb{1}-\bar{P}_{e}\left(\bar{L}_{0}(\omega)-e\right)^{-1} \bar{P}_{e} I(\omega)\right] P_{e} \eta_{e}^{(s, r)} \otimes \psi_{\mathrm{R}}+O\left(N^{2}\left(\lambda_{1}^{2}+\lambda_{2}^{2}\right)\right) .
$$

Proceeding in the same way we get the following representation for the eigenvectors $\widetilde{\chi}_{e}^{(s, r)}$ of the adjoint operator $\left[K_{\lambda_{1}, \lambda_{2}}(\omega)\right]^{*}$,

$$
\tilde{\chi}_{e}^{(s, r)}=\left[\mathbb{1}-\bar{P}_{e}\left(\bar{L}_{0}(\bar{\omega})-e\right)^{-1} \bar{P}_{e}\left(I^{*}\right)(\bar{\omega})\right] P_{e} \widetilde{\eta}_{e}^{(s, r)} \otimes \psi_{\mathrm{R}}+O\left(N^{2}\left(\lambda_{1}^{2}+\lambda_{2}^{2}\right)\right),
$$


where $\widetilde{\eta}_{e}^{(s, r)}$ satisfies (2.2). Relations (3.15) and (3.16) give

$$
\chi_{e}^{(s, r)}=\eta_{e}^{(s, r)} \otimes \psi_{\mathrm{R}}+O\left(N\left(\left|\lambda_{1}\right|+\left|\lambda_{2}\right|\right)\right), \quad \tilde{\chi}_{e}^{(s, r)}=\widetilde{\eta}_{e}^{(s, r)} \otimes \psi_{\mathrm{R}}+O\left(N\left(\left|\lambda_{1}\right|+\left|\lambda_{2}\right|\right)\right),
$$

with the additional properties $\left\{\mathbb{1} \otimes\left\langle\psi_{\mathrm{R}}\right|\right\} \chi_{e}^{(s, r)}=\eta_{e}^{(s, r)} \otimes \psi_{\mathrm{R}}+O\left(N^{2}\left(\lambda_{1}^{2}+\lambda_{2}^{2}\right)\right)$ and $\left\{\mathbb{1} \otimes\left\langle\psi_{\mathrm{R}}\right|\right\} \widehat{\chi}_{e}^{(s, r)}=\widetilde{\eta}_{e}^{(s, r)} \otimes \psi_{\mathrm{R}}+O\left(N^{2}\left(\lambda_{1}^{2}+\lambda_{2}^{2}\right)\right)$.

Relations (3.10) and (3.17) show that

$$
\begin{aligned}
& \left\langle\psi_{0}, B^{\prime} Q_{e}^{(s)}\left[A \otimes \mathbb{1}_{\mathrm{S}} \otimes \mathbb{1}_{\mathrm{R}}\right] \psi_{\mathrm{ref}}\right\rangle= \\
& \quad \sum_{r=1}^{R}\left\langle\psi_{0}, B^{\prime} \eta_{e}^{(s, r)} \otimes \psi_{\mathrm{R}}\right\rangle\left\langle\widetilde{\eta}_{e}^{(s, r)} \otimes \psi_{\mathrm{R}},\left[A \otimes \mathbb{1}_{\mathrm{S}} \otimes \mathbb{1}_{\mathrm{R}}\right] \psi_{\mathrm{ref}}\right\rangle+O\left(N^{2}\left(\lambda_{1}^{2}+\lambda_{2}^{2}\right)\right) .
\end{aligned}
$$

Let us take $A=\left|\varphi_{\underline{\tau}}\right\rangle\left\langle\varphi_{\underline{\sigma}}\right|$. Then we have (see also after (3.2))

$$
\left\langle\widetilde{\eta}_{e}^{(s, r)},\left[A \otimes \mathbb{1}_{\mathrm{S}}\right] \psi_{\mathrm{S}, \infty}\right\rangle=2^{-N / 2}\left\langle\widetilde{\eta}_{e}^{(s, r)}, \varphi_{\underline{\tau}, \underline{\sigma}}\right\rangle .
$$

Next we insert a decomposition of identity, and use again the explicit form of $\psi_{\text {ref }}$, (3.2), to obtain

$$
\begin{aligned}
\left\langle\psi_{0}, B^{\prime} \eta_{e}^{(s, r)} \otimes \psi_{\mathrm{R}}\right\rangle & =\sum_{\underline{\sigma}^{\prime}, \underline{\tau}^{\prime}}\left\langle\psi_{0}, B^{\prime} \varphi_{\underline{\sigma}^{\prime}, \underline{\tau}^{\prime}} \otimes \psi_{\mathrm{R}}\right\rangle\left\langle\varphi_{\underline{\sigma}^{\prime}, \underline{\tau}^{\prime}}, \eta_{e}^{(s, r)}\right\rangle \\
& =2^{N / 2} \sum_{\underline{\sigma}^{\prime}, \underline{\tau}^{\prime}}\left\langle\psi_{0}, B^{\prime}\left[\left|\varphi_{\underline{\sigma}^{\prime}}\right\rangle\left\langle\varphi_{\underline{\tau}^{\prime}}\right| \otimes \mathbb{1}_{\mathrm{S}} \otimes \mathbb{1}_{\mathrm{R}}\right] \psi_{\mathrm{ref}}\right\rangle\left\langle\varphi_{\underline{\sigma}^{\prime}, \underline{\tau}^{\prime}}, \eta_{e}^{(s, r)}\right\rangle \\
& =2^{N / 2} \sum_{\underline{\sigma}^{\prime}, \underline{\tau}^{\prime}}\left\langle\varphi_{\underline{\sigma}^{\prime}, \underline{\tau}^{\prime}}, \eta_{e}^{(s, r)}\right\rangle\left[\bar{\rho}_{0}\right]_{\underline{\underline{\tau}}^{\prime}, \underline{\sigma}^{\prime}} .
\end{aligned}
$$

In the last step above, we commute $B^{\prime}$ to the right, $\left\langle\psi_{0}, B^{\prime}\left[\left|\varphi_{\sigma^{\prime}}\right\rangle\left\langle\varphi_{\tau_{\tau^{\prime}}}\right| \otimes \mathbb{1}_{\mathrm{S}} \otimes \mathbb{1}_{\mathrm{R}}\right] \psi_{\text {ref }}\right\rangle=$ $\left\langle\psi_{0},\left[\left|\varphi_{\underline{\sigma}^{\prime}}\right\rangle\left\langle\varphi_{{\underline{\tau^{\prime}}}^{\prime}}\right| \otimes \mathbb{1}_{\mathrm{S}} \otimes \mathbb{1}_{\mathrm{R}}\right] \psi_{0}\right\rangle=\left[\bar{\rho}_{0}\right]_{{\underline{\tau^{\prime}}}^{\prime} \underline{\sigma}^{\prime}}$. Equations (3.18)-(3.20) demonstrate Theorem 3.3. This also concludes the proof of Theorem 2.1.

\subsection{Proof of Theorem 2.2}

The level shift operator associated to an eigenvalue $e$ of $L_{\mathrm{S}}$ is defined as

$$
\Lambda_{e}=-P_{e} I \bar{P}_{e}\left(\bar{L}_{0}-e+\mathrm{i} 0\right)^{-1} \bar{P}_{e} I P_{e},
$$

where $P_{e}$ is the spectral projection of $L_{0}$ associated to $\{e\}, \bar{P}_{e}=\mathbb{1}-P_{e}$ and $\bar{L}_{0}$ is the operator $L_{0}$ restricted to the range of $\bar{P}_{e}{ }^{6}{ }^{6}$ The interaction operator $I$ has the form

$$
I=V-J \Delta^{1 / 2} V J \Delta^{1 / 2}
$$

where $J$ and $\Delta$ are the modular conjugation and the modular operator associated to the pair $\left(\mathfrak{M}, \psi_{\text {ref }}\right)$, where $\psi_{\text {ref }}$ is the reference vector given in $(3.2)$, and $\mathfrak{M}$ is the von Neumann algebra of observables. The operator $V$ is given by

$$
V=\lambda_{1}\left(v_{1} \otimes \mathbb{1}_{\mathrm{S}}\right) \otimes \phi_{\beta}\left(g_{1}\right)+\lambda_{2}\left(v_{2} \otimes \mathbb{1}_{\mathrm{S}}\right) \otimes \phi_{\beta}\left(g_{2}\right) .
$$

\footnotetext{
${ }^{6}$ Relative to [16], this definition differs by a sign.
} 
Here, $v_{1}$ and $v_{2}$ are the non-demolition and energy exchange interaction operators, see (1.4), and $\phi_{\beta}(g)=\frac{1}{\sqrt{2}}\left(a_{\beta}^{*}(g)+a_{\beta}(g)\right)$ are the thermal field operators.

Theorem 3.5 (1) The level shift operator $\Lambda_{e}$ has the form

$$
\Lambda_{e}=\mathrm{i} \lambda_{1}^{2} y_{1}(e)+\lambda_{2}^{2}\left[x_{2}(e)+\mathrm{i} y_{2}(e)\right]+\sum_{\left\{j: \sigma_{j}=\tau_{j}\right\}} M^{j},
$$

where $y_{1}(e), x_{2}(e)$ and $y_{2}(e)$ are given in (2.13), (2.14) and (2.15), and where the operator $M^{j}$ is understood to act non-trivially only on the two dimensional subspace, spanned by $\left\{\varphi_{++}, \varphi_{--}\right\}$, of the $j$-th factor $\mathbb{C}^{2} \otimes \mathbb{C}^{2}$ in the Hilbert space (1.15). It is represented by the matrix

$$
M^{j}=\left[\begin{array}{cc}
a+\mathrm{i} b c & -\mathrm{i} b c \\
-\mathrm{i} b & -a+\mathrm{i} b
\end{array}\right],
$$

where $a, b$ and $c$ are given in (2.17).

(2) The eigenvalues (2.16) of $M^{j}$ satisfy $\operatorname{Im}\left(z_{j}^{ \pm}\right) \geq 0$, and they are strictly positive if $a b \neq 0$. For $a=0$, we have $z_{j}^{+}=\mathrm{i} b(c+1), z_{j}^{-}=0$ and for $b=0$, we have $z_{j}^{ \pm}= \pm a$. The eigenvectors are given by (2.21).

Since $M^{j}$ acts non-trivially on different factors of the Hilbert space for different $j$, we immediately see that the eigenvalues of $\Lambda_{e}$ are the $\delta_{e}^{\underline{(\underline{e})}},(2.11)$. This proves Theorem 2.2 .

Proof of Theorem 3.5 The level shift operators (3.21), for interaction operators $V=\lambda G \otimes \mathbb{1}_{\mathrm{S}} \otimes \phi_{\beta}(g)$ and reference states $\psi_{\mathrm{S}, \beta} \otimes \psi_{\mathrm{R}}$ (equilibrium state for the uncoupled dynamics), have been calculated explicitly in [16], Proposition 5.1. An easy adaptation to the present situation gives the following result.

Proposition 3.6 We have the decomposition $\Lambda_{e}=\lambda_{1}^{2} \Lambda_{e, 1}+\lambda_{2}^{2} \Lambda_{e, 2}$, where $\Lambda_{\#}=$ $\lim _{\epsilon \downarrow 0} \Lambda_{\#}(\epsilon)$, with

$$
\begin{aligned}
-2 \Lambda_{e, 1}(\epsilon)= & P_{e}\left(v_{1}^{2} \otimes \mathbb{1}-\mathbb{1} \otimes v_{1}^{2}\right) P_{e}\left\langle g_{1}, \frac{\omega}{\omega^{2}+\epsilon^{2}} g_{1}\right\rangle \\
& -P_{e}\left(v_{1} \otimes \mathbb{1}-\mathbb{1} \otimes v_{1}\right)^{2} P_{e}\left\langle g_{1}, \operatorname{coth}(\beta \omega / 2) \frac{\mathrm{i} \epsilon}{\omega^{2}+\epsilon^{2}} g_{1}\right\rangle,
\end{aligned}
$$

where $\omega \geq 0$ is the radial variable (spherical coordinates), and

$$
\begin{aligned}
& -2 \Lambda_{e, 2}(\epsilon) \\
& =\quad P_{e}\left(v_{2} \otimes \mathbb{1}\right) \int_{\mathbb{R} \times S^{2}} \frac{u^{2}\left|g_{2}(|u|, \Sigma)\right|^{2}}{\left|1-\mathrm{e}^{-\beta u}\right|}\left(L_{\mathrm{S}}-e+u+\mathrm{i} \epsilon\right)^{-1}\left(v_{2} \otimes \mathbb{1}\right) P_{e} \\
& \quad+P_{e}\left(\mathbb{1} \otimes v_{2}\right) \int_{\mathbb{R} \times S^{2}} \frac{u^{2}\left|g_{2}(|u|, \Sigma)\right|^{2}}{\left|1-\mathrm{e}^{+\beta u}\right|}\left(L_{\mathrm{S}}-e+u+\mathrm{i} \epsilon\right)^{-1}\left(\mathbb{1} \otimes v_{2}\right) P_{e} \\
& -P_{e}\left(v_{2} \otimes \mathbb{1}\right) \int_{\mathbb{R} \times S^{2}} \frac{u^{2}\left|g_{2}(|u|, \Sigma)\right|^{2}}{\left|1-\mathrm{e}^{-\beta u}\right|}\left(L_{\mathrm{S}}-e+u+\mathrm{i} \epsilon\right)^{-1}\left(\mathbb{1} \otimes v_{2}\right) P_{e} \\
& -P_{e}\left(\mathbb{1} \otimes v_{2}\right) \int_{\mathbb{R} \times S^{2}} \frac{u^{2}\left|g_{2}(|u|, \Sigma)\right|^{2}}{\left|1-\mathrm{e}^{+\beta u}\right|}\left(L_{\mathrm{S}}-e+u+\mathrm{i} \epsilon\right)^{-1}\left(v_{2} \otimes \mathbb{1}\right) P_{e} .
\end{aligned}
$$


All integration measures are $\mathrm{d} u \mathrm{~d} \Sigma$, where $\mathrm{d} u$ is the Lebesgue measure, and $\mathrm{d} \Sigma$ is the uniform measure on $S^{2}$.

Remark. There are no "cross-terms" involving products of $v_{1}$ and $v_{2}$ in the expression (3.21): Indeed, for instance

$$
\lambda_{1} \lambda_{2} P_{e}\left(v_{1} \otimes \mathbb{1}_{\mathrm{S}} \otimes \phi_{\beta}\left(g_{1}\right)\right) \bar{P}_{e}\left(\bar{L}_{0}-e+\mathrm{i} 0\right)^{-1} \bar{P}_{e}\left(v_{2} \otimes \mathbb{1}_{\mathrm{S}} \otimes \phi_{\beta}\left(g_{2}\right)\right) P_{e}=0,
$$

since $S_{j}^{x}$ (occurring in $v_{2}$ ) maps any eigenspace of $L_{\mathrm{S}}$ into its orthogonal complement.

Proposition 3.7 We have $\Lambda_{e, 2}=x_{2}(e)+\mathrm{i} y_{2}(e)+\mathrm{i} \Gamma_{e}$, where $x_{2}(e)$ and $y_{2}(e)$ are given in (2.14) and (2.15), and where

$$
\Gamma_{e}=\sum_{\left\{j: \sigma_{j}=\tau_{j}\right\}} \Gamma^{j}
$$

with

$$
\Gamma^{j}=4 \pi B_{j}^{2} \mathcal{G}_{2}\left(2 B_{j}\right)\left(1-F_{j} \mathrm{e}^{-2 \beta B_{j}\left(S_{j}^{z} \otimes \mathbb{1}\right)}\right)\left|1-\mathrm{e}^{-2 \beta B_{j}\left(S_{j}^{z} \otimes \mathbb{1}\right)}\right|^{-1} .
$$

The operator $\Gamma^{j}$ is understood to act non-trivially only on the two-dimensional subspace, spanned by $\left\{\varphi_{++}, \varphi_{--}\right\}$, of the $j$-th factor $\mathbb{C}^{2} \otimes \mathbb{C}^{2}$ in the Hilbert space (1.15). The "flip operator" $F_{j}$ is defined by $F_{j} \varphi_{\underline{\sigma}, \underline{\tau}}=\varphi_{\sigma_{1} \tau_{1}} \otimes \cdots \otimes \varphi_{\left(-\sigma_{j}\right)\left(-\tau_{j}\right)} \otimes \cdots \otimes \varphi_{\sigma_{N} \tau_{N}}$.

In the orthonormal basis $\left\{\varphi_{++}, \varphi_{--}\right\}$, (see also (1.9)) the operator $\Gamma^{j}$ has the form

$$
\Gamma^{j}=4 \pi \frac{B_{j}^{2} \mathcal{G}_{2}\left(2 B_{j}\right)}{\mathrm{e}^{2 \beta B_{j}}-1}\left[\begin{array}{cc}
\mathrm{e}^{2 \beta B_{j}} & -\mathrm{e}^{2 \beta B_{j}} \\
-1 & 1
\end{array}\right] .
$$

Proof. We leave out the tensor product symbols $\otimes$ when no confusion should occur. Take a $\varphi_{\underline{\sigma}, \underline{\tau}}$ in the range of $P_{e}$. It follows from

$$
\left(v_{2} \otimes \mathbb{1}\right) \varphi_{\underline{\sigma}, \underline{\tau}}=\sum_{j=1}^{N} \varphi_{\sigma_{1} \tau_{1}} \cdots \varphi_{\left(-\sigma_{j}\right) \tau_{j}} \cdots \varphi_{\sigma_{N} \tau_{N}}
$$

that

$$
\begin{aligned}
L_{\mathrm{S}}\left(v_{2} \otimes \mathbb{1}\right) \varphi_{\underline{\sigma}, \underline{\tau}} & =\sum_{j=1}^{N}\left[\sum_{k \neq j} B_{k}\left(\sigma_{k}-\tau_{k}\right)+B_{j}\left(-\sigma_{j}-\tau_{j}\right)\right] \varphi_{\sigma_{1} \tau_{1}} \cdots \varphi_{\left(-\sigma_{j}\right) \tau_{j}} \cdots \varphi_{\sigma_{N} \tau_{N}} \\
& =\sum_{j=1}^{N}\left(e-2 B_{j} \sigma_{j}\right) \varphi_{\sigma_{1} \tau_{1}} \cdots \varphi_{\left(-\sigma_{j}\right) \tau_{j}} \cdots \varphi_{\sigma_{N} \tau_{N}} .
\end{aligned}
$$

We now apply $P_{e}\left(v_{2} \otimes \mathbb{1}\right)=P_{e} \sum_{k=1}^{N}\left(S_{k}^{x} \otimes \mathbb{1}\right)$ to $(3.34)$. The only contribution is coming from terms where $k=j$ in the resulting double sum: indeed, $\left(S_{k}^{x} S_{j}^{x} \otimes \mathbb{1}\right) \varphi_{\underline{\sigma}, \underline{\tau}}$ is orthogonal to the range of $P_{e}$ unless $k=j$. It follows that

$$
(3.27) \varphi_{\underline{\sigma}, \underline{\tau}}=\int_{\mathbb{R} \times S^{2}} \mathrm{~d} u \mathrm{~d} \Sigma \frac{u^{2}\left|g_{2}(|u|, \Sigma)\right|^{2}}{\left|1-\mathrm{e}^{-\beta u}\right|} \sum_{j=1}^{N}\left(-2 B_{j} \sigma_{j}+u+\mathrm{i} \epsilon\right)^{-1} \varphi_{\underline{\sigma}, \underline{\tau}} .
$$


In particular, the operator (3.27) is diagonal in the energy basis. Proceeding in the same fashion one finds

$$
L_{\mathrm{S}}\left(\mathbb{1} \otimes v_{2}\right) \varphi_{\underline{\sigma}, \underline{\tau}}=\sum_{j=1}^{N}\left(e+2 B_{j} \tau_{j}\right) \varphi_{\sigma_{1} \tau_{1}} \cdots \varphi_{\sigma_{j}\left(-\tau_{j}\right)} \cdots \varphi_{\sigma_{N} \tau_{N}}
$$

and

$$
(3.28) \varphi_{\underline{\sigma}, \underline{\tau}}=\int_{\mathbb{R} \times S^{2}} \mathrm{~d} u \mathrm{~d} \Sigma \frac{u^{2}\left|g_{2}(|u|, \Sigma)\right|^{2}}{\left|1-\mathrm{e}^{+\beta u}\right|} \sum_{j=1}^{N}\left(2 B_{j} \tau_{j}+u+\mathrm{i} \epsilon\right)^{-1} \varphi_{\underline{\sigma}, \underline{\tau}} .
$$

The operator (3.28) is thus also diagonal in the energy basis. Next we consider (3.29). We apply $P_{e}\left(v_{2} \otimes \mathbb{1}\right)=P_{e} \sum_{k=1}^{N}\left(S_{k}^{x} \otimes \mathbb{1}\right)$ to (3.36). The only non-vanishing contribution comes from $k=j$ in the resulting double sum and only for terms where $\sigma_{j}-\tau_{j}=0$. We obtain

$$
\begin{aligned}
(3.29) \varphi_{\underline{\sigma}, \underline{\tau}}= & -\sum_{\left\{j: \sigma_{j}=\tau_{j}\right\}} \int_{\mathbb{R} \times S^{2}} \mathrm{~d} u \mathrm{~d} \Sigma \frac{u^{2}\left|g_{2}(|u|, \Sigma)\right|^{2}}{\left|1-\mathrm{e}^{-\beta u}\right|} \\
& \times\left(2 B_{j} \sigma_{j}+u+i \epsilon\right)^{-1} \varphi_{\sigma_{1} \tau_{1}} \cdots \varphi_{\left(-\sigma_{j}\right)\left(-\tau_{j}\right)} \cdots \varphi_{\sigma_{N} \tau_{N}} .
\end{aligned}
$$

Note that $\sigma_{j}$ can be replaced by $\tau_{j}$ in (3.38). A similar argument gives

$$
\begin{aligned}
(3.30) \varphi_{\underline{\sigma}, \underline{\tau}}= & -\sum_{\left\{j: \sigma_{j}=\tau_{j}\right\}} \int_{\mathbb{R} \times S^{2}} \mathrm{~d} u \mathrm{~d} \Sigma \frac{u^{2}\left|g_{2}(|u|, \Sigma)\right|^{2}}{\left|1-\mathrm{e}^{+\beta u}\right|} \\
& \times\left(-2 B_{j} \sigma_{j}+u+\mathrm{i} \epsilon\right)^{-1} \varphi_{\sigma_{1} \tau_{1}} \cdots \varphi_{\left(-\sigma_{j}\right)\left(-\tau_{j}\right)} \cdots \varphi_{\sigma_{N} \tau_{N}} .
\end{aligned}
$$

The operators (3.29) and (3.30) are not diagonal in the energy basis.

Next we use (3.27)-(3.30) and $\lim _{\epsilon \rightarrow 0_{+}}(-\alpha+u+\mathrm{i} \epsilon)^{-1}=-\mathrm{i} \pi \delta(u-\alpha)+\mathrm{P} . \mathrm{V} \cdot \frac{1}{u-\alpha}$ in (3.35) and (3.37)-(3.39) to arrive at

$$
\begin{aligned}
\Lambda_{e, 2} \varphi_{\underline{\sigma}, \underline{\tau}}= & {\left[x_{2}(e)+\mathrm{i} y_{2}(e)\right] \varphi_{\underline{\sigma}, \underline{\tau}} } \\
& +4 \mathrm{i} \pi \sum_{\left\{j: \sigma_{j}=\tau_{j}\right\}} B_{j}^{2} \frac{\mathcal{G}_{2}\left(2 B_{j}\right)}{\mid 1-\mathrm{e}^{-2 \beta B_{j} \sigma_{j} \mid}} \varphi_{\underline{\sigma}, \underline{\tau}} \\
& -4 \mathrm{i} \pi \sum_{\left\{j: \sigma_{j}=\tau_{j}\right\}} B_{j}^{2} \frac{\mathcal{G}_{2}\left(2 B_{j}\right)}{\mid 1-\mathrm{e}^{+2 \beta B_{j} \sigma_{j} \mid}} \varphi_{\sigma_{1} \tau_{1}} \cdots \varphi_{\left(-\sigma_{j}\right)\left(-\tau_{j}\right)} \cdots \varphi_{\sigma_{N} \tau_{N}}
\end{aligned}
$$

We have $\left|1-\mathrm{e}^{2 \beta B_{j} \sigma_{j}}\right|^{-1}=\frac{\mathrm{e}^{-2 \beta B_{j} \sigma_{j}}}{\mid 1-\mathrm{e}^{-2 \beta B_{j} \sigma_{j} \mid}}$, so (3.41) plus (3.42) combine to

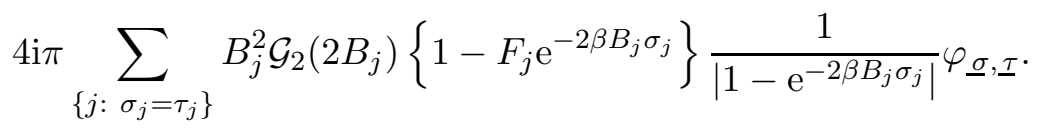

The form (3.33) of $\Gamma^{j}$ in the basis $\left\{\varphi_{++}, \varphi_{--}\right\}$is immediately obtained from (3.32). This completes the proof of Proposition 3.7.

The following result follows directly from (3.26). 
Proposition 3.8 We have

$$
\Lambda_{e, 1}=\mathrm{i} y_{1}(e)-e_{0} \mathrm{P} . \mathrm{V} \cdot\left\langle g_{1}, \omega^{-1} g_{1}\right\rangle \sum_{\left\{j: \sigma_{j}=\tau_{j}\right\}} \sigma_{j},
$$

where $y_{1}$ and $e_{0}$ are given in (2.13) and (2.18).

We obtain (3.24) now by combining Propositions 3.7 and 3.8. This shows point (1) of Theorem 3.5. Point (2) is verified easily by using the expression (2.16).

\subsection{Proof of Theorem 2.3}

According to $(1.20)$ we have $\gamma_{e}=\min \left\{\operatorname{Im} \varepsilon_{e}^{(\underline{\underline{\varrho}})}: \underline{\varrho} \in\{+1,-1\}^{N_{0}(e)}\right.$ s.t. $\left.\varepsilon_{e^{(\underline{\varrho})}} \neq 0\right\}$, where $e=e(\underline{\sigma}, \underline{\tau})=\sum_{j=1}^{N} B_{j}\left(\sigma_{j}-\tau_{j}\right)$.

- For $e \neq 0$ and $\lambda_{1}, \lambda_{2}$ satisfying (A1), we have $e+\delta_{e}^{(\underline{\underline{\varrho}})} \neq 0$, and hence $\varepsilon_{e}^{\underline{(\underline{\varrho})}} \neq 0$, if $\left|\lambda_{1}\right|,\left|\lambda_{2}\right|<c$, for some $c>0$. The smallest imaginary part of $\delta_{e}^{(\underline{\underline{e}})}$ (for $e$ fixed) is $\lambda_{1}^{2} y_{1}(e)+\lambda_{2}^{2} y_{2}(e)+y_{12}(e)$.

- For $e=0$, we have $e+\delta_{e}^{(\underline{\underline{\varrho}})}=\sum_{\left\{j: \sigma_{j}=\tau_{j}\right\}} z_{j}^{\varrho_{j}}$. Indeed, $e=0$ forces $\sigma_{j}=\tau_{j}$ for all $j$, and $e_{0}=0$ (see (2.18)). It follows that $a_{j}=0$ and so

$$
z_{j}^{+}=4 \pi \mathrm{i} \lambda_{2}^{2} B_{j}^{2} \mathcal{G}_{2}\left(2 B_{j}\right) \operatorname{coth}\left(\beta B_{j}\right) \quad \text { and } \quad z_{j}^{-}=0 .
$$

The smallest imaginary part of $\delta_{e}^{(\underline{\varrho})}$ is thus zero, corresponding to $\varrho_{j}=-1$ for all $j=1, \ldots, N_{0}(0)=N .^{7}$ All other imaginary parts are strictly larger than the gap given by $(2.23)$.

This shows formula (1.22) and completes the proof of Theorem 2.3.

\subsection{Proof of Theorem 2.5}

Let $\varphi_{\underline{\sigma}, \underline{\tau}}$ be an eigenvector of $L_{\mathrm{S}}$ associated to the eigenvalue $e(\underline{\sigma}, \underline{\tau})$. Let $k=1, \ldots, N$ be a fixed index. The vector $\left(S_{k}^{x} \otimes S_{k}^{x}\right) \varphi_{\underline{\sigma}, \tau}$ is again an eigenvector of $L_{\mathrm{S}}$ with eigenvalue $e\left(\underline{\sigma}^{\prime}, \underline{\tau}^{\prime}\right)$, where $\left(\sigma_{j}^{\prime}, \tau_{j}^{\prime}\right)=\left(\sigma_{j}, \tau_{j}\right)$ for all $j \neq k$, and $\left(\sigma_{k}^{\prime}, \tau_{k}^{\prime}\right)=\left(-\sigma_{k},-\tau_{k}\right)$. We now show that $e(\underline{\sigma}, \underline{\tau}) \neq e\left(\underline{\sigma}^{\prime}, \underline{\tau}^{\prime}\right)$ unless $e(\underline{\sigma}, \underline{\tau})=0$. Indeed, suppose that $e(\underline{\sigma}, \underline{\tau})=$ $e\left(\underline{\sigma}^{\prime}, \underline{\tau}^{\prime}\right)$. Then, due to the genericness of the parameters $J_{i j}$ and $B_{j}$ (see after $(2.27)$ ), we have $n_{k}=0$, from which it follows that $\sigma_{k}=\tau_{k}$. Furthermore, since $m_{i k}=0$ for all $i$, we obtain $\sigma_{i}=\tau_{i}$ for $i=1, \ldots, N$. We conclude that for all $k$,

$$
P_{e(\underline{\sigma}, \underline{\tau})}\left(S_{k}^{x} \otimes S_{k}^{x}\right) \varphi_{\underline{\sigma}, \underline{\tau}}=0, \quad \text { if } \quad e(\underline{\sigma}, \underline{\tau}) \neq 0,
$$

where $P_{e}$ is the spectral projection of $L_{\mathrm{S}}$ associated to $e$. One sees also easily that if $k \neq l$, then $P_{e(\underline{\sigma}, \underline{\tau})}\left(S_{k}^{x} \otimes S_{l}^{x}\right) \varphi_{\underline{\sigma}, \underline{\tau}}=0$.

\footnotetext{
${ }^{7}$ It can be inferred from general considerations that at least one eigenvalue of $\Lambda_{0}$ must be zero. Indeed, since the generator $K_{\lambda_{1}, \lambda_{2}}$ has been designed to annihilate the reference state $\psi_{\text {ref }}=\psi_{\mathrm{S}, \infty} \otimes \psi_{\mathrm{R}}$, it follows that $\Lambda_{0} \psi_{\mathrm{S}, \infty}=0$ [12]. Note that indeed, for $\varrho_{j}=-1$, all $j$, we have $\eta_{0}^{(\underline{\varrho})}=\psi_{\mathrm{S}, \infty}$.
} 
Proposition 3.9 The level shift operators $\Lambda_{e}$ with $e \neq 0$ are diagonal in the energy basis. Their eigenvalues are given by $\lambda_{1}^{2}\left[x_{1}+\mathrm{i} y_{1}\right]+\lambda_{2}^{2}\left[x_{2}+\mathrm{i} y_{2}\right]$ (see (2.12), (2.13) and (2.29), (2.30)).

Proof. The spectrum of $\Lambda_{e, 2}$ (see Proposition 3.6) is obtained as in the proof of Proposition 3.7. Relations (3.34) and (3.36) are replaced by

$$
\begin{aligned}
L_{\mathrm{S}}\left(v_{2} \otimes \mathbb{1}\right) \varphi_{\underline{\sigma}, \underline{\tau}} & =\sum_{j=1}^{N}\left[e(\underline{\sigma}, \underline{\tau})+v_{j}(\underline{\sigma}, \underline{\tau})\right] \varphi_{\sigma_{1} \tau_{1}} \cdots \varphi_{\left(-\sigma_{j}\right) \tau_{j}} \cdots \varphi_{\sigma_{N} \tau_{N}} \\
L_{\mathrm{S}}\left(\mathbb{1} \otimes v_{2}\right) \varphi_{\underline{\sigma}, \underline{\tau}} & =\sum_{j=1}^{N}\left[e(\underline{\sigma}, \underline{\tau})+v_{j}^{\prime}(\underline{\sigma}, \underline{\tau})\right] \varphi_{\sigma_{1} \tau_{1}} \cdots \varphi_{\sigma_{j}\left(-\tau_{j}\right)} \cdots \varphi_{\sigma_{N} \tau_{N}}
\end{aligned}
$$

where $v_{j}, v_{j}^{\prime}$ are given in (2.30). The terms (3.38) and (3.39) vanish due to (3.46). It then follows easily that the spectrum of $\Lambda_{e, 2}$ is $\lambda_{2}^{2}\left[x_{2}+\mathrm{i} y_{2}\right]$.

The operator $\Lambda_{e, 1}$ is the same as in the case $J_{i j}=0$, so we can again use Proposition 3.8, and, together with Proposition 3.6, this gives the result.

\section{A Dual bases, projections, resonance eigenvectors}

Proposition A.1 Let $Q$ be a finite-dimensional projection in a Hilbert space $\mathcal{H}$. Given any basis $\left\{\chi_{r}\right\}$ of $\operatorname{Ran} Q$, there is a unique basis $\left\{\widetilde{\chi}_{r}\right\}$ of $\operatorname{Ran} Q^{*}$ satisfying the duality condition $\left\langle\chi_{r}, \widetilde{\chi}_{r^{\prime}}\right\rangle=\delta_{r, r^{\prime}}$. For $\chi_{r}, \widetilde{\chi}_{r}$ obtained in this way, we have $Q=\sum_{r}\left|\chi_{r}\right\rangle\left\langle\widetilde{\chi}_{r}\right|$.

Proof. Take any basis $\left\{\chi_{r}\right\}$ of $\operatorname{Ran} Q$ and let $\psi \in \mathcal{H}$ be arbitrary. We have $Q \psi=$ $\sum_{r} \chi_{r} c_{r}(\psi)$, where $\psi \mapsto c_{r}(\psi) \in \mathbb{C}$ is a linear functional. Consequently, for each $r$, there is a $\widetilde{\chi}_{r} \in \mathcal{H}$ such that $c_{r}(\psi)=\left\langle\widetilde{\chi}_{r}, \psi\right\rangle$. Hence $Q=\sum_{r}\left|\chi_{r}\right\rangle\left\langle\widetilde{\chi}_{r}\right|$. The vector $\chi_{r^{\prime}}$ is left invariant by $Q$, so it follows that $\left\langle\widetilde{\chi}_{r}, \chi_{r^{\prime}}\right\rangle=\delta_{r, r^{\prime}}$.

We show that the $\tilde{\chi}_{r}$ are a basis of $\operatorname{Ran} Q^{*}$. Firstly, we have $Q^{*} \widetilde{\chi}_{r}=\widetilde{\chi}_{r}$ since $Q^{*}=\sum_{r}\left|\tilde{\chi}_{r}\right\rangle\left\langle\chi_{r}\right|$ and $\left\langle\tilde{\chi}_{r}, \chi_{r^{\prime}}\right\rangle=\delta_{r, r^{\prime}}$, so we only need to show linear independence. Let $z_{r}$ be scalars. If $\sum_{r} z_{r} \widetilde{\chi}_{r}=0$, then, by taking the inner product with $\chi_{r^{\prime}}$, where $r^{\prime}$ is arbitrary, and using that $\left\langle\widetilde{\chi}_{r}, \chi_{r^{\prime}}\right\rangle=\delta_{r, r^{\prime}}$, we see that $z_{r^{\prime}}=0$.

To show uniqueness of $\left\{\widetilde{\chi}_{r}\right\}$ for fixed $\left\{\chi_{r}\right\}$, we suppose that $\left\{\widetilde{\alpha}_{r}\right\}$ is another dual basis. Then $\widetilde{\alpha}_{r}=\sum_{r^{\prime}} \mu_{r, r^{\prime}} \widetilde{\chi}_{r^{\prime}}$ and by the duality condition, $\left\langle\chi_{r}, \widetilde{\alpha}_{r^{\prime}}\right\rangle=\delta_{r, r^{\prime}}=\mu_{r, r^{\prime}}$, so $\mu$ is the identity.

Proposition A.2 There are bases $\left\{\eta_{e}^{(s, r)}\right\}_{r}$ and $\left\{\widetilde{\eta}_{e}^{(s, r)}\right\}_{r}$ of the eigenspaces of $\Lambda_{e}$ and $\left(\Lambda_{e}\right)^{*}$ associated to the eigenvalue $\delta_{e}^{(s)}$ and its complex conjugate, satisfying the $d u$ ality property (2.3). Those bases are unique in the following sense: any other pair of such bases $\left\{\alpha_{e}^{(s, r)}\right\}_{r},\left\{\widetilde{\alpha}_{e}^{(s, r)}\right\}_{r}$ is given by $\alpha_{e}^{(s, r)}=\sum_{r^{\prime}}[A]_{r, r^{\prime}} \eta_{e}^{\left(s, r^{\prime}\right)}$ and $\widetilde{\alpha}_{e}^{(s, r)}=$ $\sum_{r^{\prime}}\left[\left(A^{-1}\right)^{*}\right]_{r, r^{\prime}} \widetilde{\eta}_{e}^{\left(s, r^{\prime}\right)}$, where $A$ is an invertible matrix.

Proof. From Proposition A.1 we know that we can find bases $\left\{\chi_{e}^{(s, r)}\right\}_{r}$ and $\left\{\widetilde{\chi}_{e}^{(s, r)}\right\}_{r}$ of the eigenspaces of $K_{\lambda_{1}, \lambda_{2}}(\omega)$ and its adjoint, associated to the eigenvalue $\varepsilon_{e}^{(s)}$ and 
its complex conjugate, respectively, so that $\left\langle\chi_{e}^{(s, r)}, \widetilde{\chi}_{e}^{\left(s, r^{\prime}\right)}\right\rangle=\delta_{r r^{\prime}}$. Expansions (3.15), (3.16) show that $\left\langle\eta_{e}^{(s, r)}, \widetilde{\eta}_{e}^{\left(s, r^{\prime}\right)}\right\rangle=\lim _{\lambda_{1}, \lambda_{2} \rightarrow 0}\left\langle\chi_{e}^{(s, r)}, \tilde{\chi}_{e}^{\left(s, r^{\prime}\right)}\right\rangle=\delta_{r, r^{\prime}}$.

We know from the proof of Proposition A.1 that there is a unique dual basis for fixed $\left\{\eta_{e}^{(s, r)}\right\}$. Thus, any pair of dual basis is gotten by a change of basis of one particular such pair. Let $\alpha_{e}^{(s, r)}$ be obtained by a base change matrix $A$ as in the proposition. There is a unique associated dual basis $\widetilde{\alpha}_{e}^{(s, r)}=\sum_{r^{\prime}}[B]_{r, r^{\prime}} \widetilde{\eta}_{e}^{\left(s, r^{\prime}\right)}$. It is easy to see that $\left\langle\alpha_{e}^{(s, r)}, \widetilde{\alpha}_{e}^{\left(s, r^{\prime}\right)}\right\rangle=\delta_{r, r^{\prime}}$ implies that $B=\left(A^{-1}\right)^{*}$.

\section{B Operators $K_{\lambda_{1}, \lambda_{2}}$ and $K_{\lambda_{1}, \lambda_{2}}(\omega)$}

The purpose of this Appendix is to provide some details on explicit formulas of the operators $K_{\lambda_{1}, \lambda_{2}}$ and $K_{\lambda_{1}, \lambda_{2}}(\omega)$. For more detail, we refer to [16].

Smoothed-out creation and annihilation operators are defined by

$$
a^{*}(g)=\int_{\mathbb{R}^{3}} g(k) a^{*}(k) \mathrm{d}^{3} k, \quad a(g)=\int_{\mathbb{R}^{3}} \overline{g(k)} a(k) \mathrm{d}^{3} k,
$$

for $g=g(k) \in L^{2}\left(\mathbb{R}^{3}, \mathrm{~d}^{3} k\right)$, and the field operator is given by

$$
\phi(g)=\frac{1}{\sqrt{2}}\left[a^{*}(g)+a(g)\right] .
$$

The so-called Araki-Woods representation gives the Hilbert space (GNS) representation of the infinitely extended Bose gas in thermal equilibrium $[3,13] .{ }^{8}$ The Hilbert space is given by the bosonic Fock space over the one-particle space $L^{2}\left(\mathbb{R} \times S^{2}, \mathrm{~d}^{3} k \times \mathrm{d} \Sigma\right)$,

$$
\mathcal{F}=\mathcal{F}\left(L^{2}\left(\mathbb{R} \times S^{2}, \mathrm{~d}^{3} k \times \mathrm{d} \Sigma\right)\right) .
$$

The thermal annihilation operators are

$$
a_{\beta}(f)=a\left(\sqrt{1+\mu_{\beta}(u)} \chi_{+}(u) u f(u, \sigma)\right)-a^{*}\left(\mathrm{e}^{\mathrm{i} \phi} \sqrt{\mu_{\beta}(-u)} \chi_{-}(u) u \bar{f}(-u, \sigma)\right),
$$

where $\mu_{\beta}(u)=\left(\mathrm{e}^{\beta u}-1\right)^{-1}, \chi_{ \pm}$are the indicator functions of $\mathbb{R}_{ \pm}$, and $\phi \in \mathbb{R}$ is an arbitrary phase. The $a_{\beta}^{*}(f)$ are obtained by taking the adjoint on the r.h.s. of (B.3). It is easy to see that the CCR are satisfied. The thermal field operator (B.1) is thus represented by

$$
\phi_{\beta}(f)=\frac{1}{\sqrt{2}}\left(a_{\beta}^{*}(f)+a_{\beta}(f)\right)=\frac{1}{\sqrt{2}}\left(a^{*}\left(f_{\beta}\right)+a\left(f_{\beta}\right)\right)=: \phi\left(f_{\beta}\right),
$$

for $f \in L^{2}\left(\mathbb{R}^{3}\right)$, where $f_{\beta}$ is defined in (A2), and where the $\phi$ in the r.h.s. is the field operator in $\mathcal{F}$. The equilibrium state is represented by the vacuum vector of $\mathcal{F}$,

\footnotetext{
${ }^{8}$ In this paper, we directly work in a spatially unitarily equivalent representation of the original representation, see [16] for details.
} 
$\Omega_{\mathrm{R}, \beta}=\Omega$. For a one-body operator $O$ acting on wave functions of the variables $(u, \sigma)$, we write

$$
\mathrm{d} \Gamma(O)=\int_{\mathbb{R} \times S^{2}} a^{*}(u, \sigma) O a(u, \sigma) \mathrm{d} u \mathrm{~d} \sigma .
$$

for the second quantization of $O$. The dynamics of the field is generated by

$$
L_{\mathrm{R}}=\mathrm{d} \Gamma(u),
$$

the second quantization of the operator of multiplication by $u$. We have $L_{\mathbb{R}} \Omega_{\mathbb{R}, \beta}=$ 0 , and for $z \in \mathbb{C}, \mathrm{e}^{z L_{\mathbb{R}}} \phi_{\beta}(f) \mathrm{e}^{-z L_{\mathbb{R}}}=2^{-1 / 2}\left[a_{\beta}\left(\mathrm{e}^{-\bar{z} u} f\right)+a_{\beta}^{*}\left(\mathrm{e}^{z u} f\right)\right]$, which gives the dynamics for $z=\mathrm{i} t$.

The Liouville operator $L_{\lambda_{1}, \lambda_{2}}$ acting on $\left(\mathbb{C}^{N} \otimes \mathbb{C}^{N}\right) \otimes \mathcal{F}$ is given by

$$
\begin{aligned}
L_{\lambda_{1}, \lambda_{2}} & =L_{0}+\lambda_{1} W_{1}+\lambda_{2} W_{2}, \\
L_{0} & =L_{\mathrm{S}}+L_{\mathbb{R}}=H_{\mathrm{S}} \otimes \mathbb{1}_{\mathrm{S}}-\mathbb{1}_{\mathrm{S}} \otimes H_{\mathrm{S}}+\mathrm{d} \Gamma(u), \\
W_{k} & =\sum_{j=1}^{N} S_{j}^{k} \otimes \mathbb{1}_{\mathrm{S}} \otimes \phi\left(\left(g_{k}\right)_{\beta}\right), \quad k=1,2,
\end{aligned}
$$

where we understand $S_{j}^{1}=S_{j}^{z}$ and $S_{j}^{2}=S_{j}^{x}$.

The deformation group $U(\omega)$ (see after (3.4)) is the translation group $U(\omega)=$ $\mathrm{e}^{-\mathrm{i} \omega \mathrm{d} \Gamma\left(\mathrm{i} \partial_{u}\right)}$, and the spectrally deformed Liouville operator is

$$
L_{\lambda_{1}, \lambda_{2}}(\omega)=L_{0}+\omega N+\lambda_{1} W(\omega)+\lambda_{2} W_{2}(\omega),
$$

where $N=\mathrm{d} \Gamma(\mathbb{1})$ is the number operator in $\mathcal{F}$, and where $W_{k}(\omega)=\mathrm{e}^{-\omega \mathrm{d} \Gamma\left(\partial_{u}\right)} W_{k} \mathrm{e}^{\omega \mathrm{d} \Gamma\left(\partial_{u}\right)}$ (see also (B.18)).

Definition of the operator $K_{\lambda_{1}, \lambda_{2}}$. This operator can be expressed in terms of the non-interacting Liouville operator $L_{0}$, the interaction $\lambda_{1} W_{1}+\lambda_{2} W_{2}$, see (B.7)-(B.9), and the modular data $J, \Delta$ associated to the vector $\psi_{\text {ref }},(3.2)$, and the von Neumann algebra $\mathfrak{M}=\mathcal{B}\left(\mathcal{H}_{\mathrm{S}}\right) \otimes \mathbb{1}_{\mathrm{S}} \otimes \mathfrak{M}_{\beta}$, where $\mathfrak{M}_{\beta}$ is the Weyl algebra of the Bose field (see e.g. $[6,16]) . J$ is an anti-unitary operator and $\Delta$ is a self-adjoint non-negative operator. The defining properties of $J$ and $\Delta$ are $J \Delta^{1 / 2} M \Omega_{\beta, 0}=M^{*} \Omega_{\beta, 0}$, for any $M \in \mathfrak{M}$, where $M^{*}$ is the adjoint operator of $M$. The explicit expressions are (see also $[6,16,15]$ )

$$
\begin{aligned}
J=J_{\mathrm{S}} \otimes J_{\mathrm{R}} \quad & \text { and } \Delta=\Delta_{\mathrm{S}} \otimes \Delta_{\mathrm{R}} \\
\Delta_{\mathrm{S}} & =\mathrm{e}^{-\beta L_{\mathrm{S}}}, \\
\Delta_{\mathrm{R}} & =\mathrm{e}^{-\beta L_{\mathrm{R}}} \\
J_{\mathrm{S}} \phi_{l} \otimes \phi_{r} & =\mathcal{C} \phi_{r} \otimes \mathcal{C} \phi_{l}, \\
J_{\mathrm{R}} \psi_{n}\left(u_{1}, \sigma_{1}, \ldots, u_{n}, \sigma_{n}\right) & =\mathrm{e}^{\mathrm{i} n \phi} \bar{\psi}_{n}\left(-u_{1}, \sigma_{1}, \ldots,-u_{n}, \sigma_{n}\right),
\end{aligned}
$$

where the action of the antilinear operator $\mathcal{C}$ is to take the complex conjugate of vector coordinates in the basis $\left\{\varphi_{j}\right\}_{j=1}^{N}$ of $\mathcal{H}_{\mathrm{S}}$, and $\bar{\psi}_{n}$ is the complex conjugate of $\psi_{n} \in \mathcal{F}$. Relation (B.15) shows that $J_{\mathrm{R}} a^{\#}(f(u, \sigma)) J_{\mathrm{R}}=a^{\#}\left(\mathrm{e}^{\mathrm{i} \phi} \bar{f}(-u, \sigma)\right)$, for $f \in L^{2}\left(\mathbb{R} \times S^{2}\right)$.

The interaction operators $I_{k}$ in (3.5) are given by $I_{k}=W_{k}-W_{k}^{\prime}$, where

$$
W_{k}^{\prime}=J \Delta^{1 / 2} W_{k} J \Delta^{1 / 2}=\mathbb{1}_{\mathrm{S}} \otimes \sum_{j=1}^{N} S_{j}^{k} \otimes \frac{1}{\sqrt{2}}\left[a^{*}\left(\left(g_{k}\right)_{\beta}\right)+a\left(\mathrm{e}^{-\beta u}\left(g_{k}\right)_{\beta}\right)\right] .
$$


The spectrally deformed operator $K_{\lambda_{1}, \lambda_{2}}(\omega)$ is obtained as follows. The transformation of creation and annihilation operators under $U(\omega),($ B.10), is

$$
U(\omega) a^{\#}(f) U(\omega)^{-1}=a^{\#}(f(\cdot+\omega)), \quad \omega \in \mathbb{R},
$$

where $f(\cdot+\omega)$ is the shifted function $(u, \sigma) \mapsto f(u+\omega, \sigma)$. Relation (B.17) can be written in the form $U(\omega) a^{\#}(f) U(\omega)^{-1}=a^{\#}\left(\mathrm{e}^{\omega \partial_{u}} f\right)$. In order to obtain an analytic extension of (B.17) to complex $\omega$, we need to take the complex conjugate of $\omega$ in the argument of the annihilation operator (since the latter is anti-linear in its argument). We thus have $I_{k}(\omega)=W_{k}(\omega)-W_{k}^{\prime}(\omega)$, with

$$
\begin{aligned}
& W_{k}(\omega)=\sum_{j=1}^{N} S_{j}^{k} \otimes \mathbb{1}_{\mathrm{S}} \otimes \frac{1}{\sqrt{2}}\left[a^{*}\left(\left(g_{k}\right)_{\beta}(\cdot+\omega)\right)+a\left(\left(g_{k}\right)_{\beta}(\cdot+\bar{\omega})\right)\right], \\
& W_{k}^{\prime}(\omega)=\mathbb{1}_{\mathrm{S}} \otimes \sum_{j=1}^{N} S_{j}^{k} \otimes \frac{1}{\sqrt{2}}\left[a^{*}\left(\left(g_{k}\right)_{\beta}(\cdot+\omega)\right)+a\left(\mathrm{e}^{-\beta(u+\bar{\omega})}\left(g_{k}\right)_{\beta}(\cdot+\bar{\omega})\right)\right] .
\end{aligned}
$$

Finally, we have $K_{\lambda_{1}, \lambda_{2}}(\omega)=L_{\lambda_{1}, \lambda_{2}}(\omega)+\lambda_{1} I_{1}(\omega)+\lambda_{2} I_{2}(\omega)$.

\section{References}

[1] Altepeter, J.B., Hadley, P.G., Wendelken, S.M., Berglund, A.J., Kwiat, P.G.: Experimental Investigation of a Two-Qubit Decoherence-Free Subspace. Phys. Rev. Lett. 92, no.14, 147901

[2] Ao, P., Rammer, J.: Quantum dynamics of a two-state system in a dissipative environment. Phys. Rev B 43, 5397-5418 (1991)

[3] Araki, H., Woods, E.J.: Representations of the canonical commutation relations describing a nonrelativistic infinite free Bose gas. Journ. Math. Phys. 4 637-662 (1963)

[4] Berman, G.P., Kamenev, D.I., Tsifrinovich, V.I.: Collective decoherence of the superpositional entangled states in the quantum Shor algorithm. Phys. Rev. A 71, 032346 (2005)

[5] Bach, V., Fröhlich, J., Sigal, I.M.: Renormalization Group Analysis of Spectral Problems in Quantum Field Theory. Adv. Math. 137, 205-298 (1998)

[6] Bratteli, O., Robinson, D.W., Operator algebras and quantum statistical mechanics. I and II, Springer Verlag 1987

[7] Duan, L.-M., Guo, G.-C.: Scheme for reducing collective decoherence in quantum memory. Phys. Lett. A 243, 265-269 (1998)

[8] Fedorov, A., Fedichkin, L.: Collective decoherence of nuclear spin clusters. J. Phys. Condens. Matter 18, 3217-3228 (2006) 
[9] Fröhlich, J., Merkli, M.: Another Return of 'Return to Equilibrium'. Comm. Math. Phys. 2003

[10] Jakšić, V., Pillet, C.-A.: Non-equilibrium steady states of finite quantum systems coupled to thermal reservoirs. Comm. Math. Phys. 226, no.1, 131-162 (2002)

[11] Jakšić, V., Pillet, C.-A.: On a model for quantum friction II. Fermi's golden rule and dynamics at positive temperature. Comm. Math. Phys. 176, no.3, 619-644 (1996)

[12] Merkli, M.: Level shift operators for open quantum systems. J. Math. Anal. Appl. 327, no. 1, 376-399 (2007)

[13] Merkli, M.: The ideal quantum gas. Open quantum systems I, Lecture Notes in Math. 1880, 183-233, Springer, Berlin, 2006.

[14] Merkli, M., Mück, M., Sigal, I.M.: Instability of Equilibrium States for Coupled Heat Reservoirs at Different Temperatures. J. Funct. Anal. 243 no. 1, 87-120 (2007)

[15] Merkli, M., Mück, M., Sigal, I.M.: Theory of Non-Equilibrium Stationary States as a Theory of Resonances. Ann. Henri Poincaré 8, 1539-1593 (2007)

[16] Merkli, M., Sigal, I.M., Berman, G.P.: Resonance Theory of Decoherence and Thermalization Annals of Physics 323, 373-412 (2008) and Decoherence and thermalization Phys. Rev. Lett. 98, no. 13, 130401 (2007)

[17] Palma,G.M., Suominen, K.-A., Ekert, A.K.: Quantum Computers and Dissipation. Proc. R. Soc. Lond. A 452, 567-584 (1996)

[18] Paz, J.P., Roncaglia, A.J.: Dynamics of the entanglement between two oscillators in the same environment, preprint, arXiv:0801.0464v1 [quant-ph] 3 Jan 2008

[19] Sorensen, A., Duan, L.-M., Cirac, J.I., Zoller, P.: Many-particle entanglement with Bose-Einstein condensates, Nature 409, 4 January 2001, 63-66

[20] Utsunomiya, S., Master, C.P., Yamamoto, Y.: Algorithm-Based Analysis of Collective Decoherence in Quantum Computation, arXiv:quant-ph/0408162v1, 2004 